\title{
Revealing the full biosphere structure and versatile metabolic functions in the deepest ocean sediment of the Challenger Deep
}

\author{
Ping Chen ${ }^{1 \dagger}$, Hui Zhou ${ }^{1,2+}$, Yanyan Huang ${ }^{3,4+}$, Zhe Xie ${ }^{5 \dagger}$, Mengjie Zhang ${ }^{1,2+}$, Yuli Wei ${ }^{5}$, Jia Li ${ }^{1,2}$, Yuewei Ma ${ }^{3}$,
} Min Luo ${ }^{5}$, Wenmian Ding ${ }^{3}$, Junwei Cao ${ }^{5}$, Tao Jiang ${ }^{1,2}$, Peng Nan ${ }^{3^{*}}$, Jiasong Fang ${ }^{5^{*}}$ and Xuan $\mathrm{Li}^{1,2^{*}}$

\author{
* Correspondence: nanpeng@fudan. \\ edu.cn; jsfang@shou.edu.cn; lixuan@ \\ sippe.ac.cn \\ 'Ping Chen, Hui Zhou, Yanyan \\ Huang, Zhe Xie and Mengjie Zhang \\ contributed equally to this work. \\ ${ }^{3}$ Ministry of Education Key \\ Laboratory for Biodiversity Science \\ and Ecological Engineering, Schoo \\ of Life Sciences, Fudan University, \\ Shanghai, China \\ ${ }^{5}$ Shanghai Engineering Research \\ Center of Hadal Science and \\ Technology, College of Marine \\ Sciences, Shanghai Ocean \\ University, Shanghai, China \\ ${ }^{1}$ CAS-Key Laboratory of Synthetic \\ Biology, CAS Center for Excellence \\ in Molecular Plant Sciences, Institute \\ of Plant Physiology and Ecology, \\ Chinese Academy of Sciences, \\ Shanghai, China \\ Full list of author information is \\ available at the end of the article
}

\begin{abstract}
Background: The full biosphere structure and functional exploration of the microbial communities of the Challenger Deep of the Mariana Trench, the deepest known hadal zone on Earth, lag far behind that of other marine realms.

Results: We adopt a deep metagenomics approach to investigate the microbiome in the sediment of Challenger Deep, Mariana Trench. We construct 178 metagenome-assembled genomes (MAGs) representing 26 phyla, 16 of which are reported from hadal sediment for the first time. Based on the MAGs, we find the microbial community functions are marked by enrichment and prevalence of mixotrophy and facultative anaerobic metabolism. The microeukaryotic community is found to be dominated by six fungal groups that are characterized for the first time in hadal sediment to possess the assimilatory and dissimilatory nitrate/sulfate reduction, and hydrogen sulfide oxidation pathways. By metaviromic analysis, we reveal novel hadal Caudovirales clades, distinctive virus-host interactions, and specialized auxiliary metabolic genes for modulating hosts' nitrogen/sulfur metabolism. The hadal microbiome is further investigated by large-scale cultivation that cataloged 1070 bacterial and 19 fungal isolates from the Challenger Deep sediment, many of which are found to be new species specialized in the hadal habitat.

Conclusion: Our hadal MAGs and isolates increase the diversity of the Challenger Deep sediment microbial genomes and isolates present in the public. The deep metagenomics approach fills the knowledge gaps in structure and diversity of the hadal microbiome, and provides novel insight into the ecology and metabolism of eukaryotic and viral components in the deepest biosphere on earth.
\end{abstract}

Keywords: Mariana Trench, Challenger Deep, Hadal sediment, Metagenomics, Versatile metabolism, Metavirome, Large-scale cultivation

\section{Background}

The hadal trench represents the deepest habitat for living organisms on the surface of the earth and accounts for a significant portion of the global benthic area [1]. In

(c) The Author(s). 2021 Open Access This article is licensed under a Creative Commons Attribution 4.0 International License, which permits use, sharing, adaptation, distribution and reproduction in any medium or format, as long as you give appropriate credit to the original author(s) and the source, provide a link to the Creative Commons licence, and indicate if changes were made. The images or other third party material in this article are included in the article's Creative Commons licence, unless indicated otherwise in a credit line to the material. If material is not included in the article's Creative Commons licence and your intended use is not permitted by statutory regulation or exceeds the permitted use, you will need to obtain permission directly from the copyright holder. To view a copy of this licence, visit http://creativecommons.org/licenses/by/4.0/. The Creative Commons Public Domain Dedication waiver (http://creativecommons.org/publicdomain/zero/1.0/) applies to the data made available in this article, unless otherwise stated in a credit line to the data. 
addition to elevated hydrostatic pressure (60-110 MPa), the trench environments are characterized as near-freezing temperatures, total darkness, poor nutrient availability, and isolation in topography [2]. Despite the harsh conditions, abundant microorganisms and metabolic activities were found to exist in both the hadal water columns and hadal sediments [3-5]. Hadal trenches were proposed to comprise specialized biodiversity related to their geographic isolation and unique environment characteristics [2]. The microbial abundance in hadal trenches was related to the availability of sedimentary organic matter, which reached the deep hadal environments by sinking via the funneling effect and by occasional landslides induced by deep ocean earthquakes [4-6].

A great deal of effort has been focused on the Mariana Trench system in the Western Pacific ocean where two tectonic plates, the Philippine Sea plate and the Pacific plate, collide $[7,8]$. It contains the deepest habitat known on earth, the Challenger Deep, $11,000 \mathrm{~m}$ below the ocean surface [9]. Characterization of the microbial species in the hadal habitat began in the 1950s using culture-based techniques [10-12]. Recent technological advances using $16 \mathrm{~S}$ ribosomal RNA (rRNA) gene profiling expanded studies on ocean microbes by circumventing the limitation of culture dependency. Investigation on microbiome from abyssal water to bottom sediment in the Mariana Trench via the $16 \mathrm{~s}$ rRNA gene profiling found that Proteobacteria, Bacteroidetes, Actinobacteria, Gemmatimonadetes, Thaumarchaeota, and Planctomycetes were dominant in the Mariana Trench habitats $[4,13,14]$. In a comparison of microbes between Mariana and Kermadec trench habitats, they comprised cosmopolitan taxa with different abundances, in addition to some autochthonous microbes associated with unique and rare OTUs $[15,16]$.

However, our understanding of the hadal microbial diversity and association of environmental factors has largely relied on the technology of $16 \mathrm{~s}$ rRNA gene profiling, and the studies of limited number of cultured microbes. It was suggested that genetic and phenotypic details of trench microbial communities would ultimately require whole metagenome studies, but not just 16S rRNA gene analyses [16]. Despite the advance in sequencing technology, few high-throughput metagenomics studies on the microbial communities in the Challenger Deep sediment habitat have been conducted. Knowledge gaps remain about its biosphere structure, and details are sparse on the metabolic functions of different microbial components that drive the biogeochemical processes in the deepest habitat. Furthermore, currently little is known about the microeukaryotic and viral components of the hadal sediment biosphere, and essential questions about their community functions and ecological importance remain unanswered.

In the current study, a twofold strategy was adopted to investigate the microbial community structure and metabolic functions in the Challenger Deep sediment, the deepest habitat on the earth. First, a deep metagenomics approach was designed and employed, by preparing extensive metagenomic libraries for Illumina sequencing, to achieve unprecedented coverage depth on its metagenome. This strategy is necessary to capture microbes of low abundances and unravel the full community structure of the hadal biosphere, underpinning its eukaryotic and viral components. It enabled us to reconstruct the largest dataset of metagenomic assembled genomes (MAGs) and to identify the versatile metabolic functions of the hadal microbiome in great detail. Second, large-scale cultivation was conducted to isolate microorganisms from the Challenger Deep sediment biosphere, using twenty-four different types of media in combination with 
different culture conditions. We obtained more than 2000 microbial isolates and cataloged 1070 bacteria and 19 fungi by $16 \mathrm{~S}$ rRNA gene or nuclear ribosomal internal transcribed spacer (ITS) tag sequencing. These microbial isolates became valuable resources for further study of adaptive mechanisms in extreme habitat.

\section{Results and discussion}

\section{Hadal sediment geochemistry and deep metagenomic sequencing}

Sediment samples were collected using two deep-sea hadal landers from the seafloor (depth of 10840 meters) at the Challenger Deep (142 $\left.21.7806^{\prime} \mathrm{E}, 11^{\circ} 25.8493^{\prime} \mathrm{N}\right)$. The sediment samples were dissected into three depth segments, i.e., the surficial segment MT-1 (sediment depth 0-5 cm), the mid-segment MT-2 (5-10 cm), and the deep segment MT-3 (10-14 cm). Geochemical measurements on the samples were taken either onboard the ship ZhangJian or inland laboratories using preserved samples. The contents of total organic carbon (TOC) and total nitrogen (TN) in the sediment were measured between 0.49 and 0.55 (wt\%) and between 0.05 and 0.06 (wt\%), respectively (Additional file 1: Table S1). They are within the previously reported values for the sediment of the Challenger Deep [13]. The values of $\delta^{13} \mathrm{C}(-21.41$ to $-21.53 \%)$ and $\delta^{15} \mathrm{~N}$ (5.42 to $6.69 \%$ ) were within the ranges of the commonly observed values for marine organic matter [17]. This agrees with the results of recent studies that marine algae were the dominant source of sedimentary organic matter in the southern Mariana Trench $[18,19]$. For nutrient ions, while the porewater $\mathrm{SO}_{4}{ }^{2-}$ concentrations were constant throughout the three segments, those of $\mathrm{NH}_{4}{ }^{+}, \mathrm{PO}_{4}{ }^{3-}$, and $\mathrm{NO}_{2}{ }^{-}$were trending upward with increasing sediment depth (Additional file 2: Table S2). The $\mathrm{NO}_{3}{ }^{-}$concentrations, on the other hand, decreased with depth. The dissolved major and trace elements remained relatively homogenous with little variations among the three segments (Additional file 3: Table S3 and S4).

To explore the full microbiome structure in the Challenger Deep sediment, we performed deep metagenomic sequencing on the sediment samples which was designed with enhanced sensitivity, to capture the genetic contents of microbes with low abundances. Note that we took steps to minimize the impact of sample temperature increase by swiftly processing and freezing sediment samples, preserving the big-picture characteristics of the microbiome for metagenomic analysis. Three or more independent Illumina libraries were generated for each segment, and on average $22.6 \mathrm{~Gb}$ sequence data were obtained from each library, generating a total of $248.65 \mathrm{~Gb}$ raw sequence data (Additional file 5: Table S5). Metagenomic co-assembly was carried out using all the clean data, which resulted in a metagenome of $6.65 \mathrm{~Gb}$ in total length with $\sim 6.21$ million contigs and an N50 of $\sim 1.14 \mathrm{~kb}$.

To reveal the compositions of the hadal sediment communities, taxonomic profiling analysis was performed on the metagenomics sequences using the kaiju program and the NCBI-nr library $[20,21]$. The relative sequence abundance for bacteria and archaea in the sediment accounted for $82.07 \%$ and $6.23 \%$, respectively, whereas that for microeukaryotes and viruses was $0.69 \%$ and $0.12 \%$, neither of which has been reported for the Challenger Deep sediment before. Different from previous studies based on rRNA gene PCR-tag sequencing, our approach estimated the sequence abundance values via the same workflow, generating scores directly comparable within the community scope. 
At the phylum level, the most abundant components were Proteobacteria, Chloroflexi, Actinobacteria, Thaumarchaeotathat, Planctomycetes, Firmicutes, Bacteroidetes, Gemmatimonadetes, Acidobacteria, and Gemmatimonadetes, belonging to either bacteria or archaea (Fig. 1A). Our results agree with previous studies relying on $16 \mathrm{~S}$ rRNA PCR-amplicon sequencing, in which Alphaproteobacteria, Chloroflexi, and Gemmatimonadetes were the most abundant bacteria, and Thaumarchaeota the most abundant archaea found in the Challenger Deep sediment habitat [16, 22]. However, the relative abundance of Thaumarchaeota varied largely between the different studies of the Mariana Trench habitats, ranging from 0.67 to $67 \%$ [23] and from 0.5 to $40 \%$ [22]. In a nonMariana system, the Yap Trench, while Thaumarchaeota was similarly enriched, the

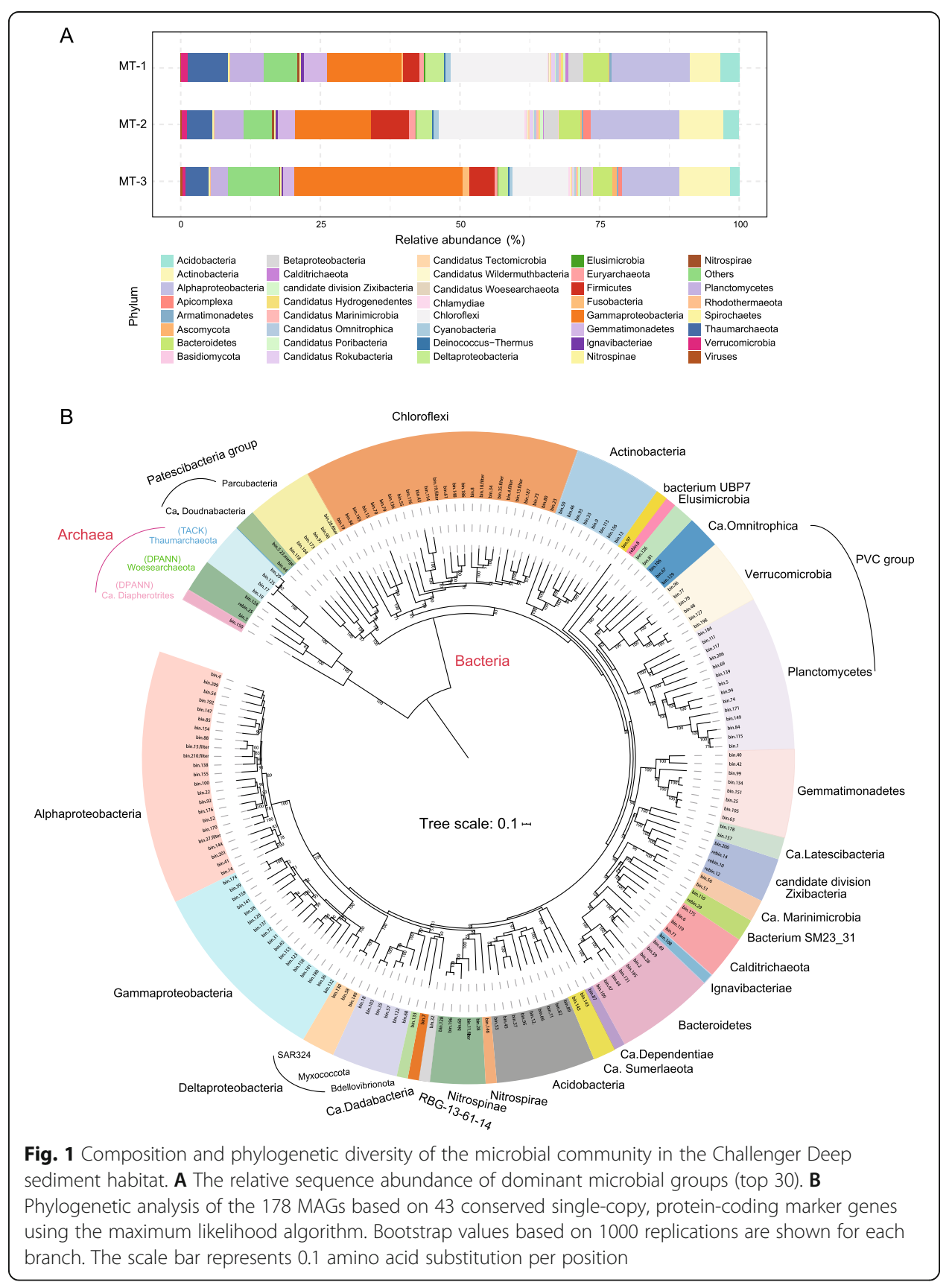


abundance of Chloroflexi, Actinobacteria, Planctomycetes, and Gemmatimonadetes in its sediment microbiome was significantly lower than that of Mariana Trench sediment habitats [24]. We found that the overall relative abundance of archaea decreased with sediment depth, similar to findings in a previous study [13]. However, the relative abundance of both the microeukaryotes and marine viruses increased with sediment depth. The most abundant eukaryotes were Opisthokonta (Fungi), Alveolata, Stramenopiles, and Rhodophyta. Note the top eukaryotic phyla, Ascomycota and Basidiomycota, ranked the 16th and 17th overall in sequence abundance (Fig. 1A). We found MT-1 and MT-2 had consistent microbial compositions compared to those of MT-3, suggesting a geochemical boundary separated them at a $10-\mathrm{cm}$ depth that was previously shown to limit oxygen access in hadal sediment [25]. Albeit in a relatively low abundance, the eukaryotic and viral components were unraveled for the first time in the Challenger Deep sediment, and as integral parts of the hadal biosphere, their ecological significance was further addressed below.

\section{Phylogenetic diversity of the hadal sediment microbiome}

Metagenomic binning on the assembled metagenome and filtering resulted in 178 quality draft genomes, i.e., MAGs (metagenome-assembled genomes) (Additional file 6: Table S6). These MAGs were at or above the medium-quality standards for draft genome, which required $>50 \%$ completeness and $<10 \%$ contamination ("Materials and method") [26]. Furthermore, among them, 101 draft genomes were $>70 \%$ completeness, and 22 were $>90 \%$ completeness. The MAGs represented the most diverse metagenome reconstructed from the hadal sediment biosphere, comprising members from 26 phyla or candidate phyla. Sixteen of them had MAGs found from the Changer Deep sediment for the first time, i.e., Gemmatimonadetes (8), Verrucomicrobia (8), Nitrospinae (5), Elusimicrobia (1), Ignavibacteriae (1), Calditrichaeota (4), candidate phyla including Zixibacteria (4), Ca. Hydrogenedentes (2), Ca. Dependentiae (1), Ca. Diapherotrites (1), Ca. Doudnabacteria (2), Ca. Latescibacteria (2), Ca. Omnitrophica (3), Ca. Marinimicrobia (2), and unclassified bacteria including Bacterium SM23_31 (2), Ca. Sumeriaeota (2) and a newly defined phylum UBP7_A (1) [27]. In comparison, previous studies reported eleven and thirty MAGs that were co-assembled by combing water and sediment microbial sequences from the Mariana Trench and were affiliated with three and twelve phyla, respectively [28, 29]. Besides, using the single-cell sequencing approach, twelve single-cell amplified genomes (SAGs) were generated for Parcubacteria from the Mariana Trench sediment [30]. Note that these earlier works had fewer MAGs despite co-assembly by mixing sample data from multiple sources. Thus, the deep metagenomics approach has significantly enhanced the coverage and sensitivity of the hadal microbiome.

To illustrate the taxonomic diversity of the hadal sediment microbes, a phylogenetic tree for the 178 MAGs was constructed (Fig. 1B), using 43 conserved single-copy, protein-coding marker genes [31,32]. A substantial number of uncultured microbial lineages were uncovered and classified with the phylogenetic analysis. The main bacterial groups included Alphaproteobacteria (22 MAGs), Gammaproteobacteria (19), Chloroflexi (19), Planctomycetota (14), Bacteroidota (10), Actinobacteriota (10), Gemmatimonadetes (8), Verrucomicrobia (6), Nitrospinae (4), as well as Elusimicrobia (1), 
Ignavibacteriae (1), Nitrospirae (1), and Calditrichaeota (4). Notably, bin.97 formed a monophyletic clade in the phylogenetic tree and was distant from the PVC groups that include Planctomycetes (Fig. 1B). It was closely related to Planctomycetales bacterium 4484_113 based on analysis using GTDB-Tk [33], for which the Average Nucleotide Identity (ANI) to bacterium 4484_113 stood at 63.86\%. Bacterium 4484_113 was originally defined as unclassified Planctomycetes, but a recent study separated it from Planctomycetes to form a new phylum UBP7_A [27]. Thus, our study uncovered a likely second member of UBP7_A from the Challenger Deep sediment habitat.

The main archaea groups included Ca. Woesearchaeota (3 MAGs), Thaumarchaeota (4), and Ca. Diapherotrites (1) (Fig. 1B). Note that Ca. Diapherotrites, represented by bin.150, was found for the first time in the Challenger Deep sediment. Intriguingly, bin.150 was situated as an outgroup to both Thaumarchaeota (TACK group) and Woesearchaeota, and had a closer relationship with bacteria in the phylogenetic tree (Fig. 1B). Based on analysis using GTDB-Tk, bin.150 was close to the unclassified Diapherotrites archaeon UBA493 that is affiliated to DPANN group. Its ANI to UBA493, the closest relative, was $72.19 \%$. We placed bin. 150 with additional members of Ca. Diapherotrites for phylogenetic analysis and found bin.150 formed a long-branch that represents a specialized Diapherotrites clade in the Challenger Deep sediment (Additional file 17: Fig. S1). A previous study reported that as a member of Ca. Diapherotrites, Ca. Iainarchaeum acquired anabolic genes from bacteria via horizontal gene transfer [34]. The same reason may explain why bin.150 had a close relationship with bacteria in our phylogenetic results. So by reconstructing the largest metagenome of the hadal sediment biosphere, we recovered representative genomes of all major prokaryotic lineages previously identified by $16 \mathrm{~S}$ rRNA gene amplicon-based surveys for the Challenger Deep sediment habitat [35], providing valuable references for us to further look into details of hadal microbiome regarding genetic diversity, metabolic functions, and symbiotic relationship. While our analyses were based on constructed MAGs, we acknowledged the missing pathway components that are likely attributed in part by the incomplete assembly, and the many assembled sequences of unknown molecular functions that are knowledge gaps to be bridged with new means [36].

\section{Versatile metabolic function of the hadal sediment microbiome Heterotrophy vs. autotrophy}

To investigate the metabolic potential of each component, the 178 MAGs reconstructed from the Challenger Deep sediment microbiome were assigned with metabolic functions based on KEGG annotation. To determine microbes' lifestyle, the MAGs were first investigated for heterotrophic potential and were found to contain genes involved in various pathways for degradation of carbohydrates (all MAGs), hydrocarbons (34 MAGs affiliated with 9 phyla), and aromatic compounds (146 MAGs affiliated with 24 phyla) (Fig. 2 and Additional file 7: Table S7). It is likely that sinking particulates from the upper ocean or terrestrial inputs, partly due to the funneling effect and earthquake-inducing landslides, were the source of the organic matter in the deepest habitat [37].

On the other hand, we found 69 out of 178 MAGs (39\%; 16 phyla) contained pathways for inorganic carbon fixation. Six different $\mathrm{CO}_{2}$ fixation mechanisms were found to involve various microbes (Fig. 2). Among them, 3-hydroxypropionate bi-cycle (3-HP) 


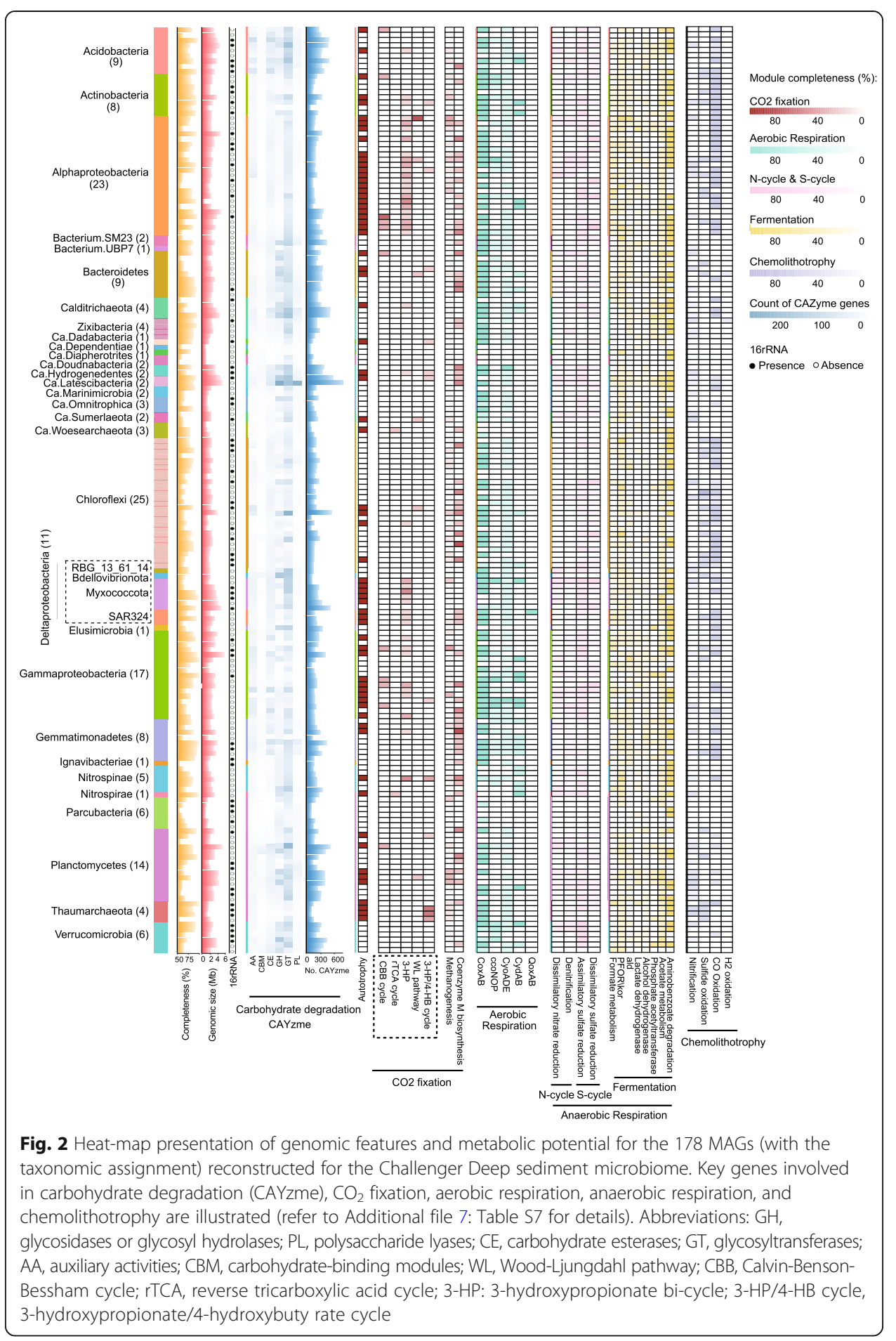

was the most prevalent in 43 MAGs, compared to the Calvin-Benson-Bassham Cycle (CBB or Calvin cycle) (11 MAGs), Wood-Ljungdahl pathway (WL pathway) (6 MAGs), the reverse TCA cycle (rTCA) (2 MAGs), the 3 hydroxypropionate-4 hydroxybutyrate cycle (3-HP/4-HB cycle) (6 MAGs), and methanogenesis (16 MAGs). Previously, 3-HP was found in Chloroflexaceae, Alpha- and Gammaproteobacteria, and SAR202 [38, 39]. In our study, the 43 MAGs detected to have the 3-HP pathway were taxonomically 
assigned to eight phyla (31\%) (Fig. 2 and Additional file 7: Table S7). They include the unclassified Chloroflexi bacterium RBG_16_64_32, Alpha-, Delta-, and Gammaproteobacteria, Nitrospinae, Acidobacteria, Actinobacteria, Calditrichaeota, Ca. Hydrogenedentes, and Gemmatimonadetes.

For other $\mathrm{CO}_{2}$ fixation pathways, the key enzymes in the Calvin Cycle, ribulosebisphosphate carboxylase $(r b c S)$, and phosphoribulokinase ( $p r k B)$ were detected in five phyla (Fig. 2 and Additional file 7: Table S7). The rTCA cycle biomarker Acl was present in one MAG for Nitrospira and one MAG for Ca. Woesearchaeota. Further, rTCA cycle was the only $\mathrm{CO}_{2}$ fixation pathway employed by Nitrospira and Ca. Woesearchaeota. While nitrite-oxidizing Nitrospira was previously known to use rTCA cycle for $\mathrm{CO}_{2}$ fixation [40], this is the first reported case that $\mathrm{Ca}$. Woesearchaeota may be capable of rTCA reaction. 3-HP/4-HB cycle was the most energy-efficient aerobic autotrophic pathway for $\mathrm{CO}_{2}$ fixation [41]. The biomarker enoyl-CoA hydratase/3-hydroxyacyl-CoA dehydrogenase involved in 3-HP/4-HB or DC/4-HB cycle was found in three bacterial groups, Nitrospinae, Acidobacteria, and Planctomycetes, and one archaea, Thaumarchaeota. The WL pathway biomarkers, i.e., acetyl-CoA decarbonylase/synthase complex subunit delta $(c d h D)$, acetyl-CoA synthase ( $a c s B)$, anaerobic carbon monoxide dehydrogenase ( $c o o S$ and $c o o F$ ), and 5-methyltetrahydrofolate corrinoid/iron-sulfur protein methyltransferase (acsE) were found in Bacteroidetes, Alphaproteobacteria, Ca. Sumerlaeota, Chloroflexi, Gammaproteobacteria, and Planctomycetes. Moreover, we found 16 MAGs associated with 7 phyla, i.e., Proteobacteria, Bacteroidetes, Chloroflexi, Gemmatimonadetes, Nitrospinae, Planctomycetes, and Thaumarchaeota, contained the Coenzyme $M$ biosynthesis and methanogenesis genes (Fig. 2 and Additional file 7: Table S7), indicating the hadal microorganisms had the potential to produce methane from fixing $\mathrm{CO}_{2}$ in the hadal zone.

Overall, among the 178 MAGs reconstructed for the hadal sediment microbiome, 69 MAGs ( 39\%) were affiliated with 16 phyla and mixotrophic based on their capacity of inorganic carbon fixation (Fig. 2 and Additional file 7: Table S7). The enrichment and significant taxonomic expansion of mixotrophic microbes we revealed for the deepest hadal microbiome may indicate an adaptive niche that mixotrophy confers to microbes living in an oligotrophic habitat like the Challenger Deep sediment.

\section{Aerobic vs anaerobic respiration}

We investigated the hadal microbiome for its potential to carry out aerobic or anaerobic respiration. In total, 155 MAGs $(\sim 87 \%)$ were found to contain aerobic respiratory genes, such as Cytochrome c oxidases (Cox/Cyd/Qox/cco/Cyo). These MAGs are associated with 24 phyla, such as Acidobacteria, Bacteroidetes, Gemmatimonadetes, and Proteobacteria (Fig. 2 and Additional file 7: Table S7). Therefore, a large majority of the microbes in the hadal habitat can potentially use oxygen as an electron acceptor for energy generation.

On the other hand, anaerobic respiration pathways were also found to be widely distributed, for which nitrate/nitrite or sulfate was the alternative electron acceptors. Dissimilatory nitrate reduction to ammonia pathway (DNRA), denitrification pathway, and sulfate reduction pathways were searched and found in a total of 145 MAGs (81\%) (Fig. 2 and Additional file 7: Table S7). These MAGs covered 21 of the 26 phyla we identified in the hadal microbiome. Specifically, the genes for DNRA (nirB and nirD as markers), denitrification pathway (nirK and norC), assimilatory sulfate reduction 
pathway (sat, $\operatorname{apr} A B$, and $d s r A B$ ), and dissimilatory sulfate reduction pathway (aprAand $a p r B$ ) were present in 64 MAGs (15 phyla), 77 MAGs (16 phyla), 135 MAGs (20 phyla), and 67 MAGs (15 phyla), respectively (Fig. 2 and Additional file 7: Table S7). Previous studies based on $16 \mathrm{~S}$ rRNA gene sequencing analysis suggested that dissimilatory nitrate reduction and denitrification were the dominant reactions, whereas microbial sulfate reduction was negligible in the Challenger Deep sediment [13]. However, our analysis shows that the broad distribution of both assimilatory sulfate reduction and dissimilatory sulfate reduction points to the equal importance of sulfate reduction pathways for anaerobic respiration, if not otherwise more important. In addition, for microbes capable of anaerobic respiration, many were found to have the potential for different types of fermentation for degradation of organic matters (Additional file 17: Analysis of fermentation; Additional file 7: Table S7).

The enrichment of anaerobic or anaerobic respiration metabolism in microbial community was apparently driven by the local habitat conditions. Examples of the deep petroleum seep sediments and Guaymas Basin hydrothermal sediments illustrated MAGs enriched for anaerobic respiration in the presence of hydrocarbons, i.e., alkanes and aromatic compounds, or depleted oxygen levels in sediments [31, 42]. We further investigated the MAGs for the potential to carry out both aerobic and anaerobic respiration and found 136 MAGs out of $178(\sim 76 \%)$, affiliated with 20 phyla (Fig. 2 and Additional file 7: Table S7), were capable of facultative anaerobic metabolism. Compared to the microbial communities in deep petroleum seeps or Guaymas Basin hydrothermal habitat, the high proportion of facultative anaerobes in the Challenger Deep sediment, reflects the microbial metabolic versatility and the ability to adapt by the hadal microbes to endemic habitats or environmental disturbance.

\section{Chemolithotrophy}

Microbes are known to acquire their energy for growth and $\mathrm{CO} 2$ fixation from the oxidation of inorganic compounds, such as hydrogen $\left(\mathrm{H}_{2}\right)$, hydrogen sulfide $\left(\mathrm{H}_{2} \mathrm{~S}\right)$, ammonia $\left(\mathrm{NH}_{3}\right)$, carbon monoxide $(\mathrm{CO})$, and metals [43]. The nitrification gene markers were found in 53 MAGs $(30 \%, 17$ phyla) of the Challenger Deep sediment microbiome (Fig. 2 and Additional file 7: Table S7). The key enzyme in nitrite oxidation, nitrite oxidoreductase/nitrate reductase was present in 35 MAGs (20\%; 13 phyla), suggesting that nitrification is an important approach for energy production for a substantial proportion of microbes in the hadal sediment biosphere. Other genes involved in nitrification pathways, $P m o A$-amoA, $P m o B-a m o B$, and $P m o C$ - $a m o C$, were detected in 4 MAGs associated with 3 phyla (Additional file 7: Table S7). Hydroxylamine dehydrogenase gene (hao) was detected in 21 MAGs (12\%; 12 phyla). Interestingly, the gene, hao, was not found in bathypelagic microbes capable of nitrification [44], reflecting the difference in microbial mediators between the bathypelagic and hadal zones in similar biogeochemical process.

The genes for $\mathrm{CO}$ oxidation, sulfide oxidation, $\mathrm{H}_{2}$ oxidation, and iron oxidation were also detected in the Challenger Deep sediment microbiome. Carbon monoxide dehydrogenase (CODH; cox gene) was found in 92 MAGs (52\%; 15 phyla) (Fig. 2 and Additional file 7: Table S7), indicating its broad distribution, and the importance of CO-oxidation as an energy source in the hadal habitat. Sulfide oxidation genes such as 
$s q r$ and $f c c B$ were found in 33 MAGs (19\%; 8 phyla), i.e., Acidobacteria, Actinobacteria, Bacteroidetes, Chloroflexi, Gemmatimonadetes, Planctomycetes, Thaumarchaeota, and Proteobacteria. For iron oxidation, we detected cyc2 gene (encoding Cytochrome c) in one MAG associated with Gemmatimonadetes, suggesting that $\mathrm{Fe}^{2+}$ can serve as an alternative electron donor for Gemmatimonadetes.

\section{Microeukaryotic community and predominant fungal groups}

Microbial eukaryotes in hadal sediment were the least studied and remained largely unknown. Our analysis found that Opisthokonta (Fungi) were the predominant eukaryotes in the Challenger Deep sediment, accounting for $87.42 \%$ of the total eukaryotic sequences. Ascomycota (41.73\%), Basidiomycota (26.82\%), and Mucoromycota (12.64\%) were the top phyla, followed by Chlorophyta (6.61\%), Rhodophyta (2.44\%), Bacillariophyta (2.31\%), Chytridiomycota (2.21\%), Zoopagomycota (1.91\%), Apicomplexa (0.96\%), etc. (Fig. 3A). Our results revealed the presence of Stramenopiles, e.g., Blastocladiomycota, and Alveolata, e.g., Apicomplexa, two members of the super-group SAR (i.e., Stramenopiles, Alveolata, and Rhizaria), in the hadal sediment, albeit at low abundances. Rhizaria was, however, notably absent. Previous studies showed that the abundance of Alveolata and Stramenopiles decreased with depth in the water column of the Mariana Trench, whereas Opisthokonta had an inverse trend [45]. Our study expanded the findings into the trench sediment, illustrating the co-existence pattern of Alveolata, Stramenopiles, and Opisthokonta.

As the dominant eukaryotic group, fungi in the Challenger Deep sediment biosphere comprised six phyla, i.e., Zoopagomycota, Mucoromycota, Ascomycota, Basidiomycota, Chytridiomycota, and Blastocladiomycota, which can be further classified into twentyseven classes (Fig. 3B). Fungi were known to be distributed globally in deep-sea water, including that in the Mariana Trench, as Basidiomycota and Ascomycota were the primary components of the deep-sea fungal community [46, 47]. Basidiomycota and Ustilaginomycetes were previously detected in deep-sea sediment using a cloning library method [48]. The capability of living in anoxia conditions may be crucial for fungi to adapt to the hadal sediment environments, as Ascomycetes, Basidiomycetes, and Chytridiomycetes were reportedly capable of fermentation and anaerobic growth in the deep ocean [49].

\section{Metabolic functions of the fungal community in the hadal sediment biosphere}

The details of gene contents and metabolic functions of the fungal community in both the water column and deep-sea sediment remained largely unknown, as early studies were based on 18S rRNA gene/ ITS amplicon sequencing [45, 47]. In the current work, we explored the metabolic potential of the fungal groups using the assembled metagenome.

\section{Carbon metabolism}

We investigated the potential of the hadal fungi to metabolize carbohydrates and peptides in the hadal sediment by looking into genes for carbohydrate-active enzymes (CAZYmes) and peptidases. In total, we detected 262 CAZYmes (88 family) and 703 peptidases (77 family) (Fig. 3B and Additional file 8: Table S8). Members of CAZyme 


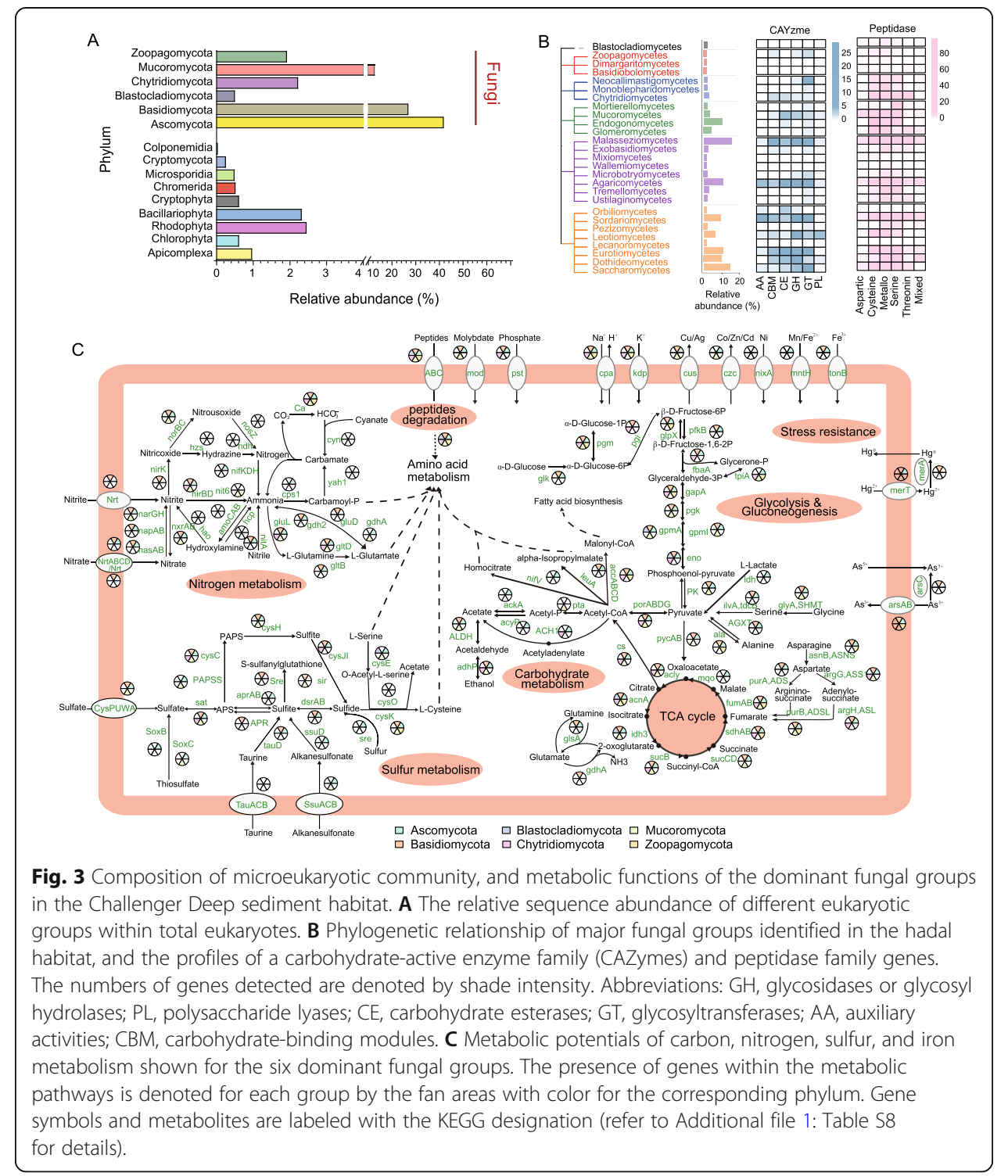

included 79 GHs (glycosidases or glycosyl hydrolases), 8 PLs (polysaccharide lyases), 51 CEs (carbohydrate esterases), 83 GTs (glycosyltransferases), 14 AAs (auxiliary activities; associated with polysaccharide and lignin degradation), and 27 CBM (carbohydratebinding modules). The major sources of CAZymes were Eurotiomycetes (number of genes $\mathrm{n}=20)$, Agaricomycetes $(\mathrm{n}=16)$, Sordariomycetes $(\mathrm{n}=38)$, and Malasseziomycetes $(\mathrm{n}=30)$. Particularly, key CAZyme genes encoding xylanase (GH10) were found in Agaricomycetes, and those encoding cellulase (GH5) were found in Agaricomycetes, Chytridiomycetes, and Microbotryomycetes (Additional file 8: Table S8).

Peptidase genes were abundant in Endogonomycetes $(\mathrm{n}=124)$, Agaricomycetes $(\mathrm{n}=$ 84), Sordariomycetes $(n=70)$, and Malasseziomycetes $(n=67)$ (Fig. 3B and Additional file 8: Table S8). Six peptidase groups, i.e., Aspartic (A), Cysteine (C), Metallo (M), Mixed (P), Serine (S), and Threonine (T) peptidases, were found in the fungal genomes. Among them, the most abundant was Metallo peptidase, consisting of 230 members that belong to 30 families, followed by Cysteine ( $\mathrm{n}=225 ; 21$ families), Serine $(\mathrm{n}=168$; 
19 families), Threonine ( $\mathrm{n}=43 ; 3$ families), and Aspartic peptidases $(\mathrm{n}=14 ; 3$ families) (Fig. 3B and Additional file 8: Table S8). So, the hadal fungi have broad potentials in organic carbon cycling, capable of degrading a variety of carbohydrate and peptide substrates.

\section{Nitrogen metabolism}

- The hadal sediment fungi were found to possess the complete pathways for dissimilatory nitrate reduction and assimilatory nitrate reduction, and the partial pathway for nitrogen denitrification, but lacked the ability for nitrification or anammox (Fig. 3C). The key enzymes for nitrate reduction, namely NarGHI/NapAB and NirBD for dissimilatory pathway, and NR/NasAB and NIT-6 for assimilatory pathway, were found in several fungal groups, indicating dissimilatory nitrate reduction is an important function for the hadal fungi. Ammonia in hadal water could be generated from decomposition of nitrogenous organic matter [4]. However, it can be limited in hadal sediment. Therefore, the nitrate reduction by the sediment fungi can be an important source of ammonia for hadal sediment microbes.

The partial pathway for nitrogen denitrification was also found in the hadal sediment fungi, missing the Nos $Z$ gene that coverts nitrous oxide to nitrogen. Downstream of the NarGHI/NapAB genes (shared with the dissimilatory nitrate reduction pathway), the NirK and NorBC genes completed the pathway to covert nitrite to nitric oxide, and nitric oxide to nitrous oxide, which may be released into the hadal sediment environment (Fig. 3C). However, unlike the bacterial community, the fungal community appeared lacking the capability of nitrification, as some key enzymes were generally missing in nitrification and anammox pathways (Fig. 3C). Notably, denitrification pathway enzymes were found in Fusarium, Penicillium, and Aspergillus from the Challenger Deep sediment (Additional file 8: Table S8). Some Fusarium species in the deep-sea oxygenlimiting environments were reportedly capable of denitrification [50]. In other lowoxygen habitats, such as anaerobic marine sediment, salt-tolerant Penicillium and Aspergillus species were also identified to carry out the denitrification process [50]. These data illustrate the potential of the hadal fungi in nitrogen metabolism, possibly having a significant role in the ecological process in the hadal environment.

\section{Sulfur metabolism}

Sulfate reduction is one of the main anaerobic respiratory pathways that many microbes living in anaerobic conditions depend on. The hadal fungi in the Challenger Deep sediment were found to possess the complete pathways for assimilatory sulfate reduction, dissimilatory sulfate reduction, and sulfide oxidation (Fig. 3C). They were also found to contain partial pathway for the SOX system, but were incomplete for oxidation of thiosulfate. The data suggest that hadal sediment fungi-like hadal bacteria, could take part in sedimentary sulfur cycle, which has not been reported for fungal communities in deep-sea sediment.

The possession of complete sulfate reduction enzymes indicated the hadal fungi had the potential of using sulfate reduction for energy production when living under anaerobic conditions (Fig. 3C). A high copy number of genes for sulfate reduction enzymes, like Sat, $\mathrm{CysC}, \mathrm{CysH}$, and $\mathrm{CysJ}$, were detected in the hadal sediment fungi (Additional 
file 8: Table S8). The sulfate adenylyltransferase gene (sat) for catalyzing the bidirectional reactions between APS and sulfate was widely distributed in members of different phyla, e.g., Ascomycota (4 classes), Basidiomycota (3 classes), Chytridiomycota ( 2 classes), and Mucoromycota (4 classes).

In the sulfide oxidation pathway, oxidation of sulfide to sulfite was catalyzed by $\operatorname{Ds} A / B$, whose genes were detected in several classes of Basidiomycota and Ascomycota, like Malasseziomycetes and Sordariomycetes. Subsequently, sulfite was oxidized to APS by adenylylsulfate reductases. Dothideomycetes (belonging to Ascomycota) contained Apr-A gene for sulfite oxidization to APS. Agaricomycetes (belonging to Basidiomycota) had the adenylylsulfate reductase gene (glutathione), denoted as $A P R$, for the same function. Finally, APS was oxidized to sulfate by the sat-coding enzymes, which was found in Ascomycota, Basidiomycota, Chytridiomycota, and Mucoromycota. The genes encoding taurine dioxygenase $(\operatorname{tau} D)$ for converting taurine to sulfite were found in Basidiomycota and Ascomycota, like Agaricomycetes, Dothideomycetes, and Eurotiomycetes. The genes encoding alkanesulfonate monooxygenase (ssuD) for converting alkanesulfonate to sulfite were found in Leotiomycetes (belonging to Ascomycota). These enzymes would produce sulfite from an organic sulfur substrate, which in turn would be fed into the sulfur metabolic pathways.

The hadal sediment fungi were also found to possess sox genes, indicating their potential for thiosulfate/sulfide oxidization. For example, Endogonomycetes of Mucoromycota contained soxC encoding sulfane dehydrogenase subunits for oxidizing thiosulfate to sulfate. Malasseziomycetes of Basidiomycota and Sordariomycetes of Ascomycota were found to have SQOR (eukaryotic sulfide quinone oxidoreductase) genes that are involved in the first step of hydrogen sulfide metabolism to produce sulfane sulfur metabolites. A previous study reported that fungi could feed sulfate to sulfatereducing bacteria (SRB) [50]. Interestingly, we also found SRB, e.g., Desulfobacterales and Desulfuromonadales (within the class Deltaproteobacteria), and Nitrospirae (class) in the Challenger Deep sediment, which corroborates the evidence for the existence of sulfide oxidation reactions and the roles that fungi that play in sulfur cycling in the hadal sediment habitat. In addition, the hydrogen sulfide metabolisms possessed by the hadal sediment fungi would counter the accumulation of sulfide $\left(\mathrm{H}_{2} \mathrm{~S}, \mathrm{HS}^{-}\right.$, and $\left.\mathrm{S}^{2-}\right)$ that was generated by SRB in the anaerobic environment. Thus, the discovery of the critical metabolic genes in sulfur metabolism implicated the important role of hadal fungal community in sulfur cycling and energy transformation in the trench environments.

\section{Metavirome in the Challenger Deep sediment habitat}

The existence and composition of viral community in the hadal sediment habitat were an open question remaining to be answered. Our deep metagenomics approach offered a new opportunity to look into the viral components for the deepest biosphere. Viral sequence reads and their taxonomic affiliations were determined using Kaiju by mapping to the viral references from NCBI-nr database. A total of fifteen major viral families were identified in the Challenger Deep sediment (Fig. 4A). dsDNA viruses were found most frequently, which were mainly affiliated with the order Caudovirales, also known as the tailed bacteriophages [52]. 


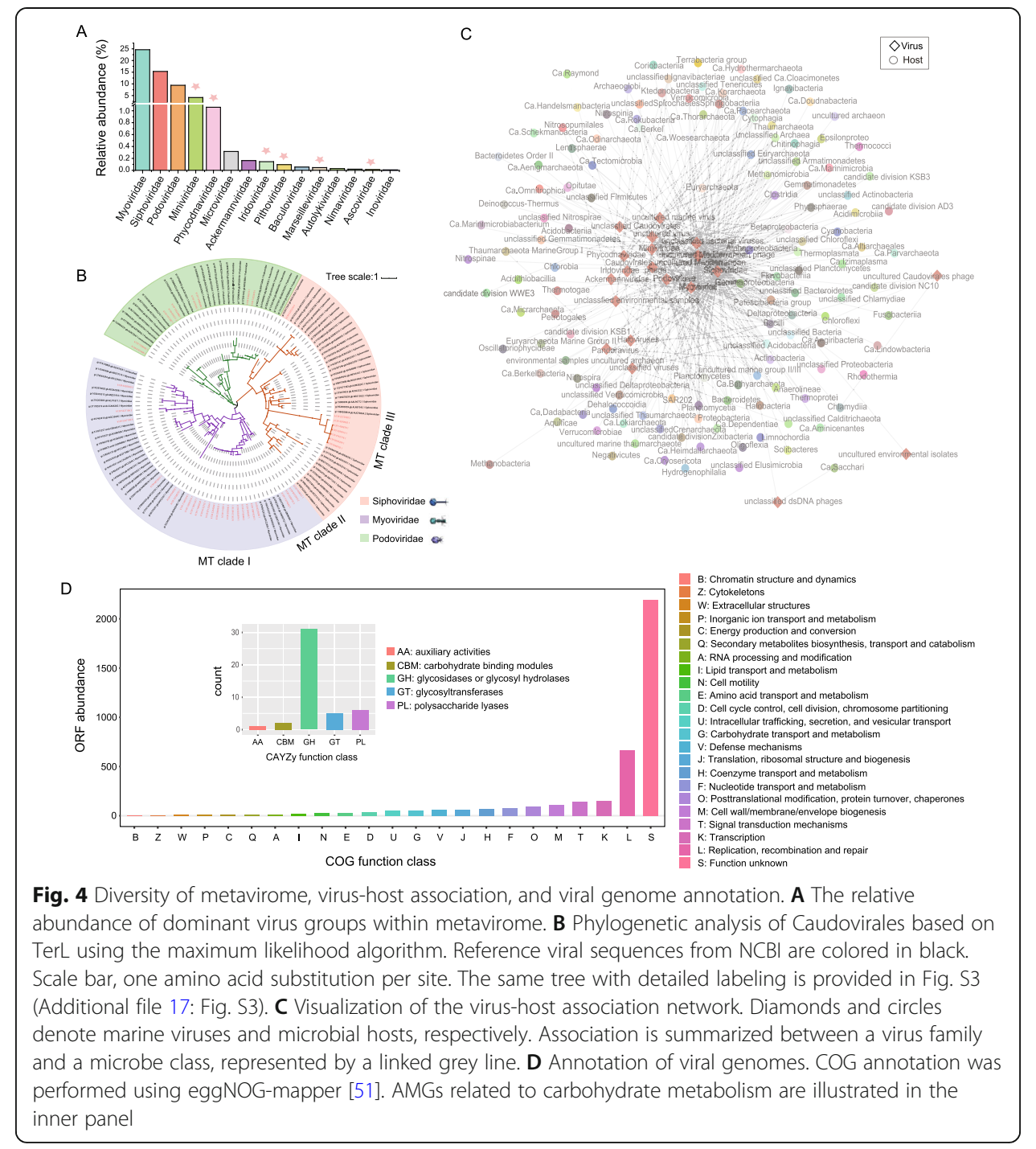

The predominant family was Myoviridae, accounting for $24.62 \%$ of the total viral sequences, followed by Siphoviridae (19.70\%) and Podoviridae (9.40\%). Notably, we detected a relatively high abundance of giant viruses in the Challenger Deep sediment, such as Mimiviridae (4.16\%) and Phycodnaviridae (1.06\%) (Fig. 4A, red star). Giant viruses were often missed or underestimated in early studies due to the technique used to capture viral particles via filtering [53]. Our approach is indifferent to the viral components of different particle sizes.

To investigate the diversity and evolution among the members of Caudovirales found in the Challenger Deep sediment, phylogenetic analysis was performed on the viral contigs, using a marker gene coding the terminase large subunit (TerL) [54]. Interestingly, for the two families, Myoviridae and Siphoviridae, there were separate branche(s) forming within each of them (Fig. 4B). They were tentatively named MT clade I and MT clade II for Myoviridae, and MT clade III for Siphoviridae. The new diversity of Caudovirales in the Challenger Deep sediment may reflect the differentiation of viral niche to microbial hosts living in the hadal habitat of high-hydrostatic pressure and oligotrophy. 


\section{Virus-host association network}

To infer virus and microbial host interactions, we took the use of a combination of different methods as described [55]. Possible virus-host interactions were summarized for each viral family. As a result, members from 131 bacteria and archaea classes were associated with 20 viral families (Fig. 4C and Additional file 8: Table S9). The association network revealed many one-to-many relationships between viruses and hosts, and vice versa. Siphoviridae was connected with the largest number of hosts that belong to 95 classes. Notably, many of the hosts were uncultured microbes that were only inferred from their presence in metagenome sequences.

Unlike in epipelagic and mesopelagic ocean waters, where the most frequent hosts were Cyanobacteria and Alphaproteobacteria (mainly SAR11 [52]), the most frequent hosts in the Challenger Deep sediment were Firmicutes (mainly Bacilli) and Bacteroidetes, followed by Euryarchaeota (including uncultured marine group II/III and Diaforarchaea), unclassified Chloroflexi, and Alphaproteobacteria. A previous study reported that the dsDNA virus T7virus (belonging to Podoviridae) could infect Deltaproteobacteria, Gammaproteobacteria, Alphaproteobacteria, Firmicutes, and Cyanobacteria [55]. Our results indicated that the T7virus from the Challenger Deep sediment was also associated with an archaeal host, e.g., the Diaforarchaea group of Euryarchaeota.

\section{Functional viromics}

To investigate the gene contents and functions of the hadal sediment viruses, we annotated their contigs using the Clusters of Orthologous Groups (COGs) database [56]. While a large proportion of their ORFs had unknown functions, high abundance genes were found in the COG categories of "replication, recombination and repair," "transcription," "signal transduction mechanism," "cell wall/membrane/envelope biogenesis," etc. (Fig. 4D). Besides the genes for basic viral functions, a special group of virusencoded genes can modulate the activities of the hosts upon infection. These so-called auxiliary metabolic genes (AMGs) were a means for viruses to manipulate host metabolism, like sulfur and nitrogen cycling.

We identified 40 putative AMGs from the Challenger Deep sediment viruses, having roles in carbon, sulfur, or nitrogen metabolism (Additional file 10: Table S10). Among them, auxiliary carbohydrate metabolic genes were the most frequent. By searching against the CAZymes database, 45 carbohydrate metabolism-related genes were identified, which included 31 GHs (glycosidases or glycosyl hydrolases), six PLs (polysaccharide lyases), five GTs (glycosyltransferases), two CBM (carbohydrate-binding modules), and one AA (auxiliary activities). The presence of frequent auxiliary carbohydrate metabolic genes provided evidence that the hadal sediment viruses may heavily manipulate carbohydrate metabolism of hosts, especially promoting carbohydrate degradation on hosts in an oligotrophic habitat. AMGs for carbohydrate metabolism were also the largest group in other marine virome dataset, like the Tara Oceans Viromes [55], which concentrated, however, on different pathways, like galactose metabolism and glycosyltransferases, reflecting the differentiated viral niche to hosts in dramatically different habitats. 
For nitrogen metabolism, NirK that encodes nitrite reductase [57] was found in the hadal viruses, suggesting possible roles to enhance host nitrite reduction pathway and release of nitric oxide into hadal sediment. For sulfur cycling, the CysN/NodQ gene encoding ATP sulphurylase [58] that reduces sulfate to produce 3'-phosphoadenosine-5'- phosphosulfate (PAPS), the first step of assimilatory sulfate reduction, was found in the hadal viruses. Notably, both NirK and CysN/NodQ are found for the first time among AMGs for ocean viruses.

\section{Large-scale isolation of microbes from the Challenger Deep sediment habitat}

To catalog and characterize microbes in the hadal sediment habitat, an intensive effort was made for the isolation of microbes from the Challenger Deep sediment. We adopted a protocol previously developed for the isolation of environmental microorganisms, which used serial dilutions of samples to allow the growth of "disadvantaged" microbes that otherwise would not be isolated [59]. To broaden the diversity of microbial isolates, we employed 24 different media and combined them with various culture conditions (Additional file 11: Table S11). Notably, the experimental conditions would enable facultative anaerobes but not strict anaerobes. As a result, more than two thousand individual isolates were obtained, for which 1089 were completed for $16 \mathrm{~S}$ rRNA gene (for prokaryotes) or ITS amplicon (for eukaryotes) sequencing (Additional file 12: Table S12 and S13).

\section{Bacterial isolates}

The taxonomy of 1070 bacterial isolates was assigned based on 16S rRNA gene sequences using SILVA database (release 123) [60]. The bacterial isolates belonged to four phyla, i.e., Proteobacteria, Bacteroidetes, Actinobacteria, and Firmicutes, which were further categorized into 7 classes, 18 orders, 25 families, and 40 genera (Additional file 17: Fig. S2 and Additional file 12: Table S12). They matched the microbes represented by sequences in the metagenome. Halomonas, Pseudoalteromonas, and Idiomarina were the top genus, accounting for $32.77 \%, 22.23 \%$, and $9.92 \%$ of the isolates, respectively (Fig. 5A). Nineteen bacterial isolates from five classes were candidates for new species, which had a $16 \mathrm{~S}$ rRNA gene sequence with $\leq 97 \%$ identity to their closest references (Additional file 14: Table S14) [61].

We characterized representative isolates from each of the four phyla, i.e., MTFD_053 (Zunongwangia sp.), MTFD_039 (Microbacterium sp.), MTFD_075 (Halomonas sp.), MTFD_0511 (Salipiger sp.), MTFD_0323 (Pseudoalteromonas sp), and MTFD_035 (Paenibacillus sp.), which were visualized after incubation at elevated pressure (100 $\mathrm{MPa}$ ) for 7 days. Some morphologic changes were observed with MTFD_039, MTFD_ 075, MTFD_0323, and MTFD_0511, when compared between their cells cultured at 0.1 and $100 \mathrm{MPa}$ (Fig. 5B). Cases were found that sister cells were connected after cell divisions under culture at elevated pressure. These isolates exemplified the small fraction of culturable microbes in contrast to the community background in the Challenger Deep sediment illustrated by the MAG data, which complemented the lack of taxonomic details from the metagenomic analysis. The results suggested that they were piezotolerant, which are likely derived from the microbes that descended from the water column, contributing to the diversity and metabolic functions of the hadal sediment microbiome. 


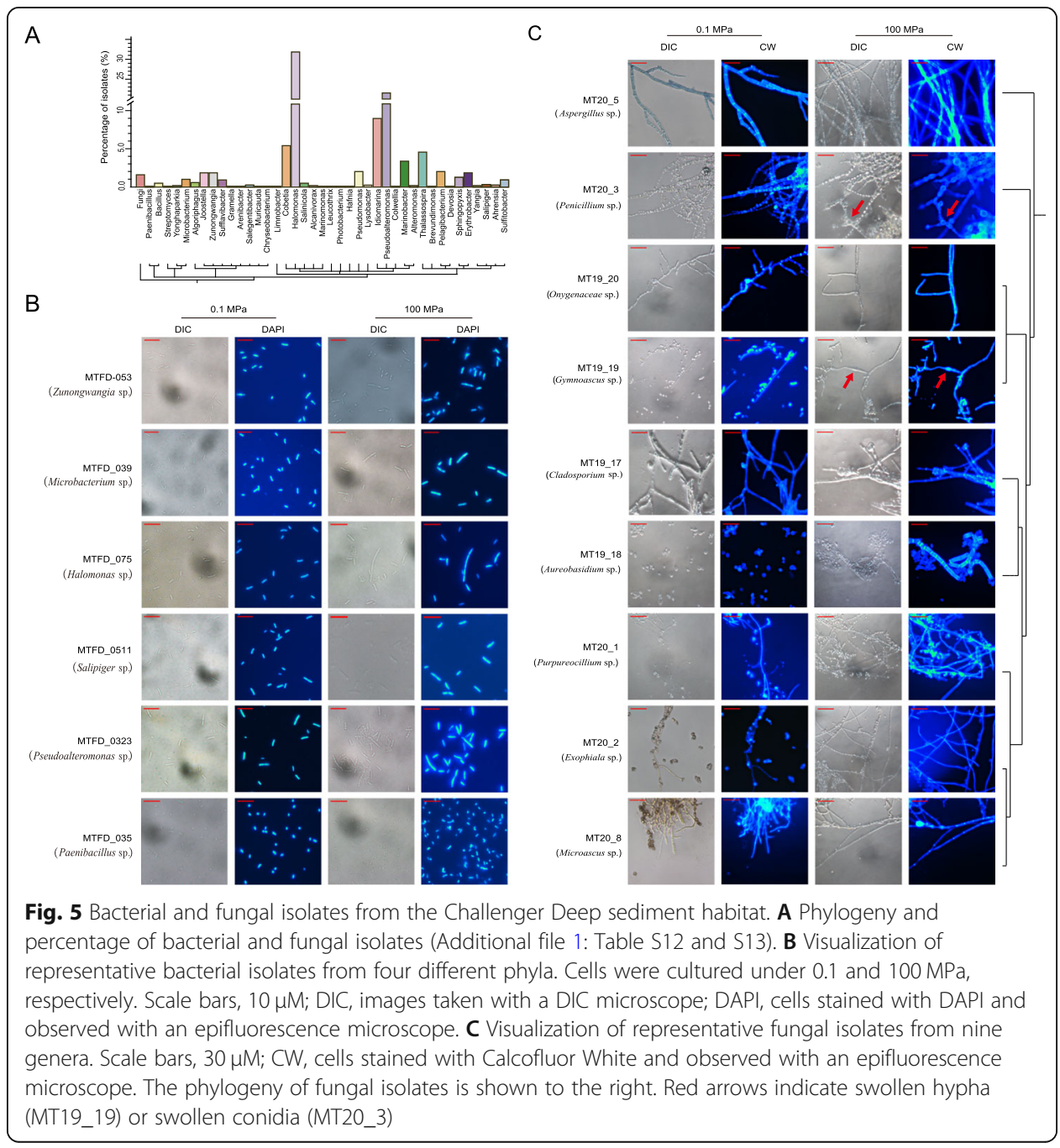

\section{Microeukaryotic isolates}

Nineteen microbial eukaryotes (fungi) were isolated from the Challenger Deep sediment (Additional file 13: Table S13 and Additional file 17: Fig. S4). Their taxonomy was assigned based on their ITS sequences using the NCBI ITS database. They belonged to nine fungal genera, namely Aspergillus, Penicillium, Acremonium, Microascus, Exophiala, Cladosporium, Gymnoascus, Purpureocillium, and Aureobasidium. They matched the eukaryotic microbes represented by sequences in the metagenome.

Representative isolates from each genus were further characterized after incubation at 0.1 and $100 \mathrm{MPa}$ for 14 days. MT19_18 (Aureobasidium sp.) tended to form long pseudomycelia under $100 \mathrm{MPa}$ (Fig. 5C). Some elongated cells contained multiple nuclei but did not have a diaphragm separating them. The filamentous fungi, i.e., MT20_2 (Exophiala sp.), MT20_5 (Aspergillus sp.), MT20_3 (Penicillium sp.), MT19-20 (Onygenaceae sp.), MT19_19 (Gymnoascus sp.), MT19_17 (Cladosporium sp.), MT20_1 (Purpureocillium sp.), and MT20_8 (Microascus sp.), have some morphologic variations induced by elevated pressure, such as hyphal swelling (MT19_19) and swollen conidia (MT20_3) (Fig. 5C). Conidiophore appeared normal for MT19_17 under $100 \mathrm{MPa}$ compared to that under 0.1 MPa. MT20_1 (Purpureocillium sp.), MT19_19 (Gymnoascus 
sp.), and MT20_8 (Microascus sp.) may represent new species specialized in the Challenger Deep habitat, whose ITS sequences diverged from their closest known species (Additional file 13: Table S13).

\section{Conclusions}

We adopted a twofold strategy to investigate the microbial community structure and functions in the Challenger Deep sediment biosphere. The deep metagenomics approach reconstructed 178 MAGs from the deepest habitat, and revealed the full biosphere structure and novel biodiversity, particularly enabling and extending our study to include microeukaryotic and viral components. The largest MAG set reconstructed for the hadal microbiome established versatile community functions marked by unexpected enrichment and prevalence of mixotrophy and facultative anaerobic metabolism. On the other hand, fungi as heterotrophs had broad metabolic potentials in carbon, nitrogen, and sulfur metabolism, particularly the capability of dissimilatory nitrate reduction and sulfate reduction that had not been reported for deep ocean microeukaryotes. These findings implicated the possible roles of hadal fungi in the biogeochemical processes of the hadal trench environment. The viral components evolved with new diversities and carried AMGs important for modulating hosts' metabolic functions, which illustrated the differentiation of viral niche to microbial hosts in the hadal habitat.

The large-scale cultivation approach obtained more than 2000 isolates from the Challenger Deep sediment, and cataloged 1070 bacteria and 19 fungi by 16 S rRNA gene or ITS amplicon sequencing. Many of them were likely new species specialized in the Challenger Deep habitat and showed morphological variations under elevated pressure $(100 \mathrm{MPa})$. These isolates represent a small fraction of the diversity in the Challenger Deep sediment habitat based on the deep metagenomic data. They would serve as model organisms and provide new opportunities to study and understand the physiology of piezotolerant microbes.

\section{Materials and methods}

\section{Sample collection and geochemical measurements}

The one intact sediment core was obtained from the seafloor in the Challenger Deep, the Mariana Trench $\left(142^{\circ} 21.7806^{\prime} \mathrm{E}, 11^{\circ} 25.8493^{\prime} \mathrm{N}, 10,840 \mathrm{~m}\right)$ during the cruise from December 2016 to January 2017 onboard the ship ZhangJian. The sediment core was collected using a box corer (with a base area of $400 \mathrm{~cm}^{2}$ and a height of $25 \mathrm{~cm}$ ) attached to the hadal lander-II, developed by Shanghai Engineering Research Center of Hadal Science and Technology, Shanghai Ocean University. After the lander reached the seafloor, the sampling chamber was slowly driven into the subseafloor until it reached around $21 \mathrm{~cm}$ below the sediment surface. A lid was then released to seal the box corer, before the lander was recovered. Once the lander surfaced and box corer was recovered and examined for integrity, only one intact sediment core with well-preserved sediment stratification was used in this study. The sediment core was immediately subsampled by inserting polycarbonate tubes to preserve the segments. The samples were either frozen at $-80^{\circ} \mathrm{C}$ before stored at $-20^{\circ} \mathrm{C}$ or stored at $4{ }^{\circ} \mathrm{C}$ for culturing and isolation of microbes. Note, the temperature of the recovered core was not obtained. For 
reference, the recorded environment temperature at the time was $2-4{ }^{\circ} \mathrm{C}$ at the ocean floor, and $27-28^{\circ} \mathrm{C}$ at the ocean surface.

Total organic carbon (TOC), total nitrogen (TN), and carbon and nitrogen isotopic compositions of particulate organic matter were measured by high-temperature combustion on a Vario Pyro Cube elemental analyzer connected to an Isoprime 100 continuous flow isotope ratio mass spectrometer. All samples were pre-treated with $10 \%$ $\mathrm{HCl}$ to remove inorganic carbon. Carbon and nitrogen isotope ratios are expressed in the delta notation $\left(\delta^{13} \mathrm{C}\right.$ and $\left.\delta^{15} \mathrm{~N}\right)$ relative to $\mathrm{V}-\mathrm{PDB}$ and atmospheric nitrogen. The average standard deviation of each measurement, determined by replicate analyses of the same sample, was $\pm 0.02 \%$ for TOC, $\pm 0.006 \%$ for $\mathrm{TN}, \pm 0.2 \%$ for $\delta^{13} \mathrm{C}$, and \pm $0.3 \%$ for $\delta^{15} \mathrm{~N}$.

For the measurement of major and minor elements, approximately $40 \mathrm{mg}$ of dry sediment powder was weighed into a Teflon beaker and dissolved in super-pure $\mathrm{HF}(0.2 \mathrm{ml}), \mathrm{HNO}_{3}(0.8 \mathrm{ml})$, and $\mathrm{HCl}(0.1 \mathrm{~mL})$. The beaker was then sealed and heated on a hot plate at $185^{\circ} \mathrm{C}$ for $36 \mathrm{~h}$. After cooling, the solution was evaporated at $120^{\circ} \mathrm{C}$ to dryness. The residue was re-dissolved by adding $2 \mathrm{ml} \mathrm{HNO}_{3}$ (superpure) and $3 \mathrm{ml}$ of deionized water at $135^{\circ} \mathrm{C}$ in an airtight beaker for $8 \mathrm{~h}$. The final solution was diluted to $50 \mathrm{ml}$ with $3 \% \mathrm{HNO}_{3}$. Blanks, duplicate samples, and several certified reference materials (GSR-1, OU-6, 1633-a, GXR-2, GXR-5) were also prepared using the same procedure. Major and minor elements were determined using a Thermo-Fisher iCAP6300 ICP-OES and a Perkin-Elmer ELAN 6000 ICPMS, respectively. The analytical precision was $5 \%$ for major and $10 \%$ for minor elements.

Measurements of $\mathrm{NO}_{2}{ }^{-}, \mathrm{NO}_{3}{ }^{-}, \mathrm{NH}_{4}{ }^{+}$, and $\mathrm{PO}_{4}{ }^{3-}$ were performed using a QuAAtro autoanalyzer (Seal Analytical) with a detection limit of $1 \mu \mathrm{M}$ and a precision of $2 \%$. Sulfate $\left(\mathrm{SO}_{4}{ }^{2-}\right)$ was measured by a Dionex ICS- $5000^{+}$ion chromatograph with a detection limit of $10 \mu \mathrm{M}$ and a precision of $2 \%$.

\section{Nucleic acid extraction and metagenomic sequencing}

DNA was extracted from $10 \mathrm{~g}$ of sediment sample for each experiment replicate, using the MoBio PowerSoil DNA Isolation kit (MO BIO Laboratories, USA) according to the manufacturer's protocol. DNA concentrations of the extract were measured with a Qubit fluorometer. For each library construction, about 100 ng DNA was fragmented with Covaris S2 (Covaris, USA) and was used to construct metagenomic DNA library with NEXTflex ${ }^{\mathrm{mm}}$ DNA Sequencing Kit compatible with the Biomek ${ }^{\circ}$ FXp (Bio Scientific, USA). Notably, PCR amplification was limited to 12 cycles for each Illumina library. The quality of DNA library was examined by Agilent Bioanalyzer 2100 (Agilent, USA) with DNA 12000 Kit. Paired-end Illumina sequencing $(2 \times 150 \mathrm{bp})$ was performed for each metagenomic library on Hiseq Xten instruments (Illumina).

\section{Metagenomic assembly, mapping, and binning}

Raw sequence data were cleaned by removing low-quality sequence reads, artificial sequence reads, and contaminated sequences with the following steps: (1) Reads with average quality score less than Q20 and a length $<30 \mathrm{bp}$, or with adapter sequences, were removed with trimmomatic (version 0.38) [62]; (2) reads with contaminated 
sequences, like plasmid sequences and human sequences, were removed with bowtie 2 (v2.3.4.1) [63]; (3) duplicated reads generated by PCR amplification were removed for metagenomic assembly using fastuniq [64]. The metagenome was assembled with clean reads using MEGAHIT (v1.1.3) [65] with the following parameters: --k-list 21, 29, 39, 59, 79, 99, 119, 127, 139. Read coverage for contigs was determined by mapping sequencing reads to contigs using bowtie 2 with default parameters.

Metagenomic binning and refinement were performed on contigs using a combination of different tools, including CONCOCT [66], Metabat [67], DAS Tool (v1.0) [68], and metaWRAP [69]. Briefly, initial binning was conducted with CONCOCT and Metabat based on tetranucleotide frequencies of contigs and coverage depth as covariance, using a contig length cutoff of 1 or $1.5 \mathrm{~kb}$, respectively, for which 310 and 400 bins were recovered. Results from the two different binning tools were then combined and merged using the DAS Tool. The resulting bins were refined and consolidated into the final bin sets using metaWRAP's Bin_refinement module with options: -c 50 -x 10. As a result, 418 bins were recovered across the entire quality spectrum. The quality of the binning results was evaluated by estimating the completeness and contamination scores using CheckM (v1.0.5) lineage_wf tool [32]. A cutoff of 50\% completeness and $10 \%$ contamination was used to filter and obtain quality genomes (MAGs).

\section{Taxonomic classification of sequences and determination of relative sequence abundance}

The method for taxonomic assignment of sequence reads and contigs was adapted from the previous work using Kaiju [20]. Briefly, the NCBI-nr database compiled in a GORG-Tropics format was downloaded, which included reference sequences from archaea, bacteria, viruses, and microbial eukaryotes. The assignment of the sequence reads or contigs was conducted using the NCBI-nr database and Kaiju (v1.7.0) [70] in Greedy-5 mode with the default options. Sequences were assigned with the taxonomic ID and functional annotations based on mapped references. Their taxonomic lineage information was then obtained using Kaiju's tool "addTaxonNames." For sequence reads assigned with taxonomic ID, the relative sequence abundance of a phylum, class, order, etc. was estimated by summarizing the total number of assigned sequence reads for a given category and dividing it with the total number of assigned reads.

\section{Phylogenetic analysis and taxonomy assignments of the metagenome-assembled genomes (MAGs)}

The sequences of 43 conserved proteins from previous work [32] were retrieved from the metagenome-assembled genomes (MAGs), and multi-aligned using the MUSCLE program (v3.8.31) [71]. The alignments were trimmed to remove gaps and poorly aligned regions using TrimAL with the options -gt 0.95 -cons 50 [72]. The "cleaned" alignment datasets for the 43 conserved proteins were then concatenated and were subsequently used for constructing a phylogenetic tree using the maximum likelihood algorithm. The RAxML program (v8.1.24) [73] was employed with the options "-f a -n boot -m PROTGAMMALG -c 4 -e 0.001 -\# 1000 ". To visualize the phylogenetic tree topology, the Newick files were processed using iTOL online tool (v4) [74]. 
Initial taxonomy assignments of MAGs were provided from the evaluation of MAGs with CheckM, which were often short with taxonomic information for the order, family, or genus. The MAGs were further classified using CAT and GTDB-Tk programs $[33,75]$. CAT assigns the taxonomy of MAGs based on the BLAST results of the $\mathrm{nr}$ database using the lowest common ancestor (LCA) algorithm [75]. On the other hand, GTDB-Tk provides taxonomic classifications with the new rank-normalized GTDB taxonomy, by using two criteria, relative evolutionary divergence (RED) and average nucleotide identity (ANI) for establishing taxonomic ranks [27, 33]. The taxonomy assignment for MAGs was determined to the deepest taxonomic levels by combining the results from both CAT and GTDB-Tk analyses. A few inconsistent assignments between the two programs were manually resolved based on information of their nearest reference neighbors.

\section{Annotation of contigs and metabolic pathway analysis}

Genes were predicted for contigs / metagenome-assembled genomes (MAGs) using prodigal (v2.6.2) with -p meta parameters [76]. Putative genes were then annotated with KEGG Automatic Annotation Server (KAAS) using the GHOSTX tool with the "custom genome dataset" and "BBH" options, by uploading their predicted amino acid sequences to KAAS [77]. Furthermore, putative genes were annotated using blastp program with default options against several different databases, like hmmer, the curated database of Anaerobic Hydrocarbon Degradation Genes (“AnHyDeg”), and MEROPS database [42]. To identify genes encoding carbohydrate degradation enzymes, the dbcan tool was used to search the Carbohydrate-Active enzymes (CAZYmes) database with an e-value cutoff of $1 \mathrm{e}-5$ [78]. To reconstruct metabolic pathways for a draft genome (MAG), its predicted genes were annotated and pathway results were summarized using the KEGG server. To illustrate metabolic activity for certain pathway(s) within a taxonomic unit (e.g., phylum, class, order) or a phylogenetic cluster, all genomes within the specific taxonomic unit/cluster were summarized, based on their taxonomic or phylogenetic assignments.

\section{Identification of viral contigs and phylogenic analysis}

Viral contigs in the metagenome assembly were identified using Kaiju (v1.7.0). For the phylogenic analysis of Caudovirales, the TerL gene [79] was identified for each contig using blastp with a threshold e-value of $10^{-5}$, minimum identity of $50 \%$, and minimum coverage of $30 \%$. Identified TerL gene sequences were retrieved from contigs and aligned using the Mafft program (version 7.407) [80]. The alignments were trimmed to remove gaps and ambiguously aligned regions before the phylogenetic tree was constructed using the Fasttree program [81], and manually formatted and visualized using iTOL.

\section{Virus-host association and viral function analysis}

Viruses and their possible hosts from the hadal sediment environment were inferred using the previously developed methods [55]. Briefly, virus-host association was predicted using three different means: (1) Virus contig-host genome similarity analysis: All identified viral contigs from the sediment of the Challenger Deep were compared to 
the archaeal and bacterial contigs in the same metagenome, using blastn with a threshold of 50 for bit score and 0.001 for E-value. (2) Virus contig-host CRISPR spacer match analysis: CRISPR spacers were predicted for all microbial contigs using MetaCRT [82]. Association between a virus and its hosts was predicted when a host CRISPR spacer was found to match a virus contig. (3) Nucleotide composition comparison between virus contigs and host genomes. The tetranucleotide-frequency vectors and mean absolute error between the vectors for each virus-host pair were computed. A viral contig was assigned to the closest microbial host whose genome having the lowest mean absolute error (d) to the viral contig if $d<0.0015$.

For functional viromics analysis, COG annotation of viral contigs was performed using eggNOG-mapper (http://eggnog-mapper.embl.de/) with a threshold of $10^{-5}$ Viral ORFs annotated as the CAZymes family genes in eggNOG-mapper annotation results were considered as auxiliary metabolic genes (AMG) related to carbohydrate metabolism [83]. Further, additional viral ORFs related to sulfur and nitrogen metabolism (determined by KEGG kos) in the eggNOG-mapper annotation results were also considered as AMGs.

\section{Large-scale cultivation and identification of the hadal sediment microbes}

The cultivation and isolation of the microbes from the Challenger Deep sediment were adopted from previous studies as described $[59,84]$. To reduce their exposure to oxygen, the sediment sub-samples were sealed in airtight bags with residue air removed before they were stored at $4{ }^{\circ} \mathrm{C}$. A series of dilutions, e.g., $10^{-1}, 10^{-2}, 10^{-3}, 10^{-4}$, and $10^{-5}$, were made to the sediment samples in 96-well plates using various culture media (Additional file 11: Table S11). The high ratio dilutions, e.g., $10^{-3}, 10^{-4}, 10^{-5}$, were then plated out in $90-\mathrm{mm}$ plates with solid culture media. The dilution and inoculation were performed under aerobic conditions in the laboratory. The plates were incubated under various conditions, i.e., $4{ }^{\circ} \mathrm{C}$ or $28{ }^{\circ} \mathrm{C}$, aerobic or anaerobic, with or without antibiotic, etc., for up to 1 month. For anaerobic culture, the agar media plates were first treated with AnaeroPack (Mitsubishi Gas Chemical Company, Inc., Tokyo) to remove oxygen. For large-scale isolation, a combination of 24 different media (modified from nine basetypes) with various culture conditions were used for cultivation (Additional file 11: Table S11). Single colonies emerging at different time points were picked to grow in liquid media for cryopreservation and further analysis. Notably some single colonies emerging at late stages were collected from the highest dilution plates, which would have been overlaid by faster-growing colonies in the low-dilution plates.

The identity of the single colonies was analyzed by $16 \mathrm{~S}$ rRNA gene (for prokaryotes) or ITS gene (for eukaryotes) sequencing. The primer sets, 8F/805R (8F-5' AGAGTTTGATCCTGGCTCAG; 805R-5'GACTACCAGGGTATCTAATC) (targeting V1-V4 regions) and 8F/1492R (8F-5' AGAGTTTGATCMTGGCTCAG; 1492R-5' GGTTACCTTGTTACGACTT) (targeting V1-V9 regions) were used for 16S rRNA gene tag PCR amplification and sequencing [85, 86]. They generated the PCR-amplified tag with length of 805 and $1492 \mathrm{bp}$, respectively. The primer set, ITS-F/ITS-R (ITS-F-5'GGAAGTAAAAGTCGTAACAAGG; ITS-R-5'TCCTCCGCTTATTGATAT GC) were used for ITS gene tag PCR amplification and sequencing. It generated the PCR-amplified tag with length of $578 \mathrm{bp}$. Identification of the sequenced single 
colony was conducted by searching $16 \mathrm{~S}$ ribosomal RNA sequence database, and Internal transcribed spacer region (ITS) database at NCBI (https://www.ncbi.nlm.nih.gov/) using blastn.

\section{Culture of microbes under elevated hydrostatic pressure and microscopic imaging}

Single colony was picked from solid media to inoculate 2-ml liquid culture media in a sterile $15-\mathrm{ml}$ glass tube and was incubated on a rotary shaker $(180 \mathrm{rpm})$ at $28^{\circ} \mathrm{C}$ overnight for bacteria or for 3 days for fungi to produce seed broth. For single-cell microbes, cultures were diluted to O.D.600 $=\sim 0.01$ in appropriate media. For filamentous fungi, mycelia in the seed broth were fragmented with sterilized scissors (if necessary) before the cultures were diluted (1/15 1/60 depending on fungal biomass) in the same media. The seeded cultures were transferred to 2-ml sterile plastic syringes before they were placed inside pressure vessels (Model: FY2016108; Feiyu Petroleum Technology Development Co., Ltd., Nantong, China). Hydrostatic pressure of $100 \mathrm{MPa}$ was applied at room temperature to bacterial cultures for 7 days, or to fungal cultures for 14 days, respectively. After high-pressure incubation, the syringes were taken out of pressure vessels and visually examined for physical integrity. The cultures were replated on solid media to test viability for growth or were examined by microscopy.

For microscopic imaging of bacteria and fungi, an epifluorescence microscope (Nikon Ecllipse 80i) and a digital camera (Nikon DS-Ri1; software: NIS-Elements F Ver4.30.01) attached to the microscope were used. For bacterial culture, cells were harvested by centrifugation. Pellets were washed twice with PBS buffer and stained with DAPI (4',6diamidino-2-phenylindole) for $10 \mathrm{~min}$ in the darkness [87]. Stained cellular suspension $(2.5 \mu \mathrm{L})$ was spread onto a class microscope slide and covered with a cover slide with excess liquid removed. Slides were imaged at $\times 100$ magnification (oil immersion len) for Differential Interference Contrast (DIC) micrograph and epifluorescent micrograph (excitation wavelength $300-380 \mathrm{~nm}$ ), respectively. For fungal culture, $10 \mu \mathrm{L}$ fungal culture was mixed with $10 \mu \mathrm{L}$ Calcofluor White solution $(1 \mathrm{mg} / \mathrm{mL})$ on a glass slide for 3 $\mathrm{min}$ at room temperature [88]. Fungal hyphae with or without Calcofluor staining were imaged at $\times 40$ magnification for DIC micrograph and epifluorescent micrograph, respectively.

\section{Supplementary Information}

The online version contains supplementary material available at https://doi.org/10.1186/s13059-021-02408-w.

Additional file 1: Table S1. Geochemical characterization of the sediment samples from Challenger Deep. Additional file 2: Table S2. Concentrations of nutrient ions $\mathrm{NO}_{3}{ }_{3}, \mathrm{NO}_{2}{ }^{-}, \mathrm{PO}_{4}{ }^{-}, \mathrm{NH}_{4}{ }^{+}$, and $\mathrm{SO}_{4}{ }^{2-}$ in porewater of the samples.

Additional file 3: Table S3. Dissolved major elements in the sediment samples.

Additional file 4: Table S4. Dissolved trace elements in the sediment samples.

Additional file 5: Table S5. Deep metagenomic sequencing on the Challenger Deep sediment samples and assembly.

Additional file 6: Table S6. Taxonomic assignment and characteristics of the 178 MAGs.

Additional file 7: Table S7. Functional analysis of archaeal and bacterial MAGs. The key genes of each metabolic pathways for MAG annotation are listed on the top. Presence/absence of genes are listed as: Presence: 1 (green); Absence: a (no color). The module completeness of each metabolic pathway is the percentage of the encoded key genes in the corresponding pathway (e.g. module completeness value of 25 means the MAG contained one of the four key genes in the pathway). 
Additional file 8: Table S8. The metabolic characteristics of the six major the Challenger Deep Fungi groups, and detailed information of the carbohydrate-active enzyme family (CAZymes) and peptidase family genes detected in major fungal groups.

Additional file 9: Table S9. List of host prediction for the Challenger Deep virome. For each prediction, the type of signal (blastn, CRISPR, tetranucleotide composition), the host sequence used for the prediction alongside its affiliation, and the strength of the prediction (length of the blastn match, number of mismatches in the CRISPR spacer, and distance between viral and host tetranucleotide frequencies vectors) are indicated.

Additional file 10: Table S10. List of eggNOG-mapper annotations for the Challenger Deep virome.

Additional file 11: Table S11. Twenty-four different media modified from several base-type that favored the growth of different bacteria or fungi.

Additional file 12: Table S12. The taxonomic assignment of the 1070 bacterial isolates. Their taxonomy was assigned based on 16S rRNA gene sequences using NCBI rRNA/ITS databases.

Additional file 13: Table S13. Characterization of nineteen sequenced Fungi.

Additional file 14: Table S14. Nineteen bacterial isolates are considered as new species, which have 165 rRNA gene sequences with $<=97 \%$ identity to closest reference.

Additional file 15: Table S15. Composition of microbial communities with other Mariana trench sites.

Additional file 16: Table S16. The relative abundance comparison of dominant microbial groups in Mariana Trench sediment with other hadal sediment samples.

Additional file 17: Supplementary Figs. S1-S4. Fig. S1. Phylogenetic analyses of bin.150 and some other Candidatus Diapherotrites archaeon reference genomes downloaded from the NCBI database. A FastTree approximate maximum likelihood phylogenetic tree was built using 15 conserved ribosomal protein. Fig. S2. Phylogenetic analyses of 1070 bacterial isolates from the hadal sediment based on the $16 \mathrm{~S}$ rRNA sequence with the FastTree. The color bar represents the order level. Fig. S3. Phylogenetic analysis of Caudovirales based on TerL using the maximum likelihood algorithm. Reference viral sequences from NCBI are colored in black. Scale bar, one amino acid substitution per site. Fig. S4. Culture of fungi under elevated hydrostatic pressure. A) High-pressure cultivation system. B) Nineteen fungi isolated from the Challenger Deep sediment, which was tested to be viable to grow on plates after subjecting to high hydrostatic pressure (100 MPa) for fourteen days. Isolate names are listed at the bottom.

Additional file 18. Analysis of MAGs on potential for different types of fermentation.

Additional file 19. Review history.

\section{Acknowledgements}

We thank the reviewers for their constructive comments which have greatly assisted us in improving this manuscript.

\section{Review history}

The review history is available as Additional file 19.

\section{Peer review information}

Anahita Bishop was the primary editor of this article and managed its editorial process and peer review in collaboration with the rest of the editorial team.

\section{Authors' contributions}

X.L. conceived the project and wrote the manuscript. J.F. and P.N. advised on the study and directed experiments for bacterial isolation and high-hydrostatic cultivation. P.C., H.Z., and J.L. conducted metagenomics experiments, analyzed data, and prepared the manuscript. Y.H., M.Z., Z.X., T.J., and W.D. performed bacterial cultivation and isolation. Y.W., J.C., and M.L. conducted geochemistry experiments and data collection. All author(s) read and approved the final manuscript.

\section{Funding}

This work was supported in part by grants from the National Key R\&D Program of China (2018YFC0310600, 2018YFA0900700), the National Natural Science Foundation of China (31771412, 91951210, 31972881, 41773069), and Biological Resources Programme from Chinese Academy of Sciences (KFJ-BRP-009).

\section{Availability of data and materials}

The metagenomic sequence data have been deposited in the NCBI Sequence Read Archive (SRA) under accession PRJNA723166 [89]. They are also available from the BioMed Big Data Center (https:/www.biosino.org/node/) under accession number OEP002357. The 16s rRNA/ITS tag sequences have been deposited to NCBI GenBank (Accession numbers listed in Tables S12 and S13).

\section{Declarations}

Ethics approval and consent to participate

Not applicable.

\section{Consent for publication}

Not applicable. 


\section{Competing interests}

The authors declare that they have no competing interests.

\section{Author details}

${ }^{1}$ CAS-Key Laboratory of Synthetic Biology, CAS Center for Excellence in Molecular Plant Sciences, Institute of Plant Physiology and Ecology, Chinese Academy of Sciences, Shanghai, China. ${ }^{2}$ University of Chinese Academy of Sciences, Beijing, China. ${ }^{3}$ Ministry of Education Key Laboratory for Biodiversity Science and Ecological Engineering, School of Life Sciences, Fudan University, Shanghai, China. ${ }^{4}$ Chinese Ancient Books reservation and Conservation Institute, Fudan University, Shanghai, China. ${ }^{5}$ Shanghai Engineering Research Center of Hadal Science and Technology, College of Marine Sciences, Shanghai Ocean University, Shanghai, China.

Received: 11 November 2020 Accepted: 10 June 2021

Published online: 13 July 2021

\section{References}

1. Jamieson AJ, Fujii T, Mayor DJ, Solan M, Priede IG. Hadal trenches: the ecology of the deepest places on Earth. Trends Ecol Evol. 2010;25(3):190-7. https://doi.org/10.1016/j.tree.2009.09.009.

2. Jamieson A. The hadal zone: life in the deepest oceans: Cambridge University Press; 2015. https://doi.org/10.1017/ CBO9781139061384.

3. Lauro FM, Bartlett DH. Prokaryotic lifestyles in deep sea habitats. Extremophiles. 2008;12(1):15-25. https://doi.org/10.1 007/s00792-006-0059-5.

4. Nunoura T, Takaki Y, Hirai M, Shimamura S, Makabe A, Koide O, et al. Hadal biosphere: insight into the microbial ecosystem in the deepest ocean on Earth. Proc Natl Acad Sci. 2015;112(11):E1230-6. https://doi.org/10.1073/pnas.142181 6112.

5. Ichino MC, Clark MR, Drazen JC, Jamieson A, Jones DO, Martin AP, et al. The distribution of benthic biomass in hadal trenches: a modelling approach to investigate the effect of vertical and lateral organic matter transport to the seafloor. Deep Sea Research Part I: Oceanographic Research Papers. 2015;100:21-33. https://doi.org/10.1016/j.dsr.2015.01.010.

6. Liu R, Wang L, Wei Y, Fang J. The hadal biosphere: recent insights and new directions. Deep Sea Research Part II: Topical Studies in Oceanography. 2018;155:11-8. https://doi.org/10.1016/j.dsr2.2017.04.015.

7. Hsui AT, Youngquist S. A dynamic model of the curvature of the Mariana Trench. Nature. 1985;318(6045):455-7. https:// doi.org/10.1038/318455a0.

8. Ohara Y, Reagan MK, Fujikura K, Watanabe H, Michibayashi K, Ishii T, et al. A serpentinite-hosted ecosystem in the Southern Mariana Forearc. Proc Natl Acad Sci. 2012;109(8):2831-5. https://doi.org/10.1073/pnas.1112005109.

9. Nakanishi M, Hashimoto J. A precise bathymetric map of the world's deepest seafloor, Challenger Deep in the Mariana Trench. Marine Geophys Res. 2011;32(4):455-63. https://doi.org/10.1007/s11001-011-9134-0.

10. Bartlett DH. Microbial life in the trenches. Marine Technol Soc J. 2009;43(5):128-31. https://doi.org/10.4031/MTSJ.43.5.5.

11. Kato C, Li L, Nogi Y, Nakamura Y, Tamaoka J, Horikoshi K. Extremely barophilic bacteria isolated from the Mariana Trench, Challenger Deep, at a depth of 11,000 meters. Appl Environ Microbiol. 1998;64(4):1510-3. https://doi.org/10.112 8/AEM.64.4.1510-1513.1998.

12. Kusube M, Kyaw TS, Tanikawa K, Chastain RA, Hardy KM, Cameron J, Bartlett DH: Colwellia marinimaniae sp. nov., a hyperpiezophilic species isolated from an amphipod within the Challenger Deep, Mariana Trench. Int I Syst Evol Microbiol 2017, 67:824-831, 4, doi: https://doi.org/10.1099/ijsem.0.001671.

13. Nunoura T, Nishizawa M, Hirai M, Shimamura S, Harnvoravongchai P, Koide O, et al. Microbial diversity in sediments from the bottom of the Challenger Deep, the Mariana Trench. Microbes Environ. 2018;33(2):186-94. https://doi.org/10.12 64/jsme2.ME17194.

14. Liu J, Zheng Y, Lin H, Wang X, Li M, Liu Y, et al. Proliferation of hydrocarbon-degrading microbes at the bottom of the Mariana Trench. Microbiome. 2019;7(1):47. https://doi.org/10.1186/s40168-019-0652-3.

15. Peoples LM, Donaldson S, Osuntokun O, Xia Q, Nelson A, Blanton J, et al. Vertically distinct microbial communities in the Mariana and Kermadec trenches. Plos one. 2018;13(4):e0195102. https://doi.org/10.1371/journal.pone.0195102.

16. Peoples LM, Grammatopoulou E, Pombrol M, Xu X, Osuntokun O, Blanton JM, et al. Microbial community diversity within sediments from two geographically separated hadal trenches. Fron Microbiol. 2019;10:347. https://doi.org/10.33 89/fmicb.2019.00347.

17. Graham M, Eaves M, Farmer J, Dobson J, Fallick A. A study of carbon and nitrogen stable isotope and elemental ratios as potential indicators of source and fate of organic matter in sediments of the Forth Estuary, Scotland. Estuarine Coastal Shelf Sci. 2001;52(3):375-80. https://doi.org/10.1006/ecss.2000.0742.

18. Luo M, Gieskes J, Chen L, Shi X, Chen D. Provenances, distribution, and accumulation of organic matter in the southern Mariana Trench rim and slope: implication for carbon cycle and burial in hadal trenches. Marine Geol. 2017;386:98-106. https://doi.org/10.1016/j.margeo.2017.02.012.

19. Guan H, Chen L, Luo M, Liu L, Mao S, Ge H, et al. Composition and origin of lipid biomarkers in the surface sediments from the southern Challenger Deep, Mariana Trench. Geosci Front. 2019;10(1):351-60. https://doi.org/10.1016/j.gsf.2018. 01.004.

20. Menzel P, Ng KL, Krogh A. Fast and sensitive taxonomic classification for metagenomics with Kaiju. Nat Commun. 2016; 7:1-9.

21. Hiraoka S, Okazaki Y, Anda M, Toyoda A, Nakano S-I, Iwasaki W. Metaepigenomic analysis reveals the unexplored diversity of DNA methylation in an environmental prokaryotic community. Nat Commun. 2019;10:159.

22. Cui G, Li J, Gao Z, Wang Y. Spatial variations of microbial communities in abyssal and hadal sediments across the Challenger Deep. PeerJ. 2019;7:e6961. https://doi.org/10.7717/peerj.6961.

23. Hiraoka S, Hirai M, Matsui Y, Makabe A, Minegishi H, Tsuda M, et al. Microbial community and geochemical analyses of trans-trench sediments for understanding the roles of hadal environments. ISME J. 2020;14(3):740-56. https://doi.org/1 0.1038/s41396-019-0564-z. 
24. Xinxu, Zhang, Wei, Xu, Yang, Liu, Mingwei, Cai, Zhuhua, Luo. Metagenomics reveals microbial diversity and metabolic potentials of seawater and surface sediment from a hadal biosphere at the Yap Trench. Front Microbiol. 2018;9:2402. https://doi.org/10.3389/fmicb.2018.02402.

25. Wenzhöfer F, Oguri K, Middelboe M, Turnewitsch R, Toyofuku T, Kitazato H, Glud RN: Benthic carbon mineralization in hadal trenches: assessment by in situ O2 microprofile measurements. Deep Sea Research Part I: Oceanographic Research Papers 2016.

26. Bowers RM, Kyrpides NC, Stepanauska R, Harmon-Smith M, Doud D, Reddy TBK, et al. Minimum information about a single amplified genome (MISAG) and a metagenome-assembled genome (MIMAG) of bacteria and archaea. Nat Biotechnol. 2017;35(8):725-31. https://doi.org/10.1038/nbt.3893.

27. Parks DH, Chuvochina M, Waite DW, Rinke C, Skarshewski A, Chaumeil P-A, et al. A standardized bacterial taxonomy based on genome phylogeny substantially revises the tree of life. Nat Biotechnol. 2018;36(10):996-1004. https://doi. org/10.1038/nbt.4229.

28. Gao ZM, Huang JM, Cui GJ, Li WL, Li J, Wei ZF, et al. In situ meta-omic insights into the community compositions and ecological roles of hadal microbes in the Mariana Trench. Environ Microbiol. 2019;21(11):4092-108. https://doi.org/1 0.1111/1462-2920.14759.

29. Huang J-M, Wang Y. Genomic differences within the phylum Marinimicrobia: from waters to sediments in the Mariana Trench. Marine Genomics. 2019;50:100699. https://doi.org/10.1016/j.margen.2019.100699.

30. León-Zayas R, Peoples L, Biddle JF, Podell S, Novotny M, Cameron J, et al. The metabolic potential of the single cell genomes obtained from the Challenger Deep, Mariana Trench within the candidate superphylum $P$ arcubacteria (OD 1). Environ Microbiol. 2017;19(7):2769-84. https://doi.org/10.1111/1462-2920.13789.

31. Dong X, Greening C, Rattray JE, Chakraborty A, Chuvochina M, Mayumi D, et al. Metabolic potential of uncultured bacteria and archaea associated with petroleum seepage in deep-sea sediments. Nat Commun. 2019;10:1-12.

32. Parks DH, Imelfort M, Skennerton CT, Hugenholtz P, Tyson GW. CheckM: assessing the quality of microbial genomes recovered from isolates, single cells, and metagenomes. Genome Res. 2015;25(7):1043-55. https://doi.org/10.1101/gr.1 86072.114

33. Chaumeil PA, Mussig AJ, Hugenholtz P, Parks DH. "GTDB-Tk: a toolkit to classify genomes with the Genome Taxonomy Database." Oxford: Bioinformatics. 2019;36(6):1925-7.https://doi.org/10.1093/bioinformatics/btz848.

34. Youssef NH, Rinke C, Stepanauskas R, Farag I, Woyke T, Elshahed MS. Insights into the metabolism, lifestyle and putative evolutionary history of the novel archaeal phylum 'Diapherotrites'. ISME J. 2015;9(2):447-60. https://doi.org/10.1038/ ismej.2014.141.

35. Nunoura T, Nishizawa M, Hirai M, Shimamura S, Harnvoravongchai $P$, Koide O, Morono Y, Fukui T, Inagaki F, Miyazaki J: Microbial diversity in sediments from the bottom of the Challenger Deep, the Mariana Trench. Microbes Environ. 2018; 33(2):ME17194. https://doi.org/10.1264/jsme2.ME17194.

36. Vanni C, Schechter MS, Acinas SG, Barberán A, Buttigieg PL, Casamayor EO, Delmont TO, Duarte CM, Eren AM, Finn RD, Kottmann R, Mitchell A, Sanchez P, Siren K, Steinegger M, Glöckner FO, Fernandez-Guerra A. bioRxiv. 2020;6(30):180448. https://doi.org/10.1101/2020.06.30.180448.

37. Jover LF, Effler TC, Buchan A, Wilhelm SW, Weitz JS. The elemental composition of virus particles: implications for marine biogeochemical cycles. Nat Rev Microbiol. 2014;12(7):519-28. https://doi.org/10.1038/nrmicro3289.

38. Zarzycki J, Brecht V, Muller M, Fuchs G. Identifying the missing steps of the autotrophic 3-hydroxypropionate CO2 fixation cycle in Chloroflexus aurantiacus. Proc Natl Acad Sci. 2009;106(50):21317-22. https:/doi.org/10.1073/pnas.0908356106.

39. Mehrshad M, Rodriguez-Valera F, Amoozegar MA, López-García P, Ghai R. The enigmatic SAR202 cluster up close: shedding light on a globally distributed dark ocean lineage involved in sulfur cycling. ISME J. 2018;12(3):655-68. https://doi.org/10.1038/s41396-017-0009-5.

40. Lucker S, Wagner M, Maixner F, Pelletier E, Koch H, Vacherie B, et al. A Nitrospira metagenome illuminates the physiology and evolution of globally important nitrite-oxidizing bacteria. Proc Natl Acad Sci U S A. 2010;107(30):1347984. https://doi.org/10.1073/pnas.1003860107.

41. Konneke M, Schubert DM, Brown PC, Hugler M, Standfest S, Schwander T, et al. Ammonia-oxidizing archaea use the most energy-efficient aerobic pathway for CO2 fixation. Proc Natl Acad Sci USA. 2014;111(22):8239-44. https://doi.org/1 $0.1073 /$ pnas.1402028111.

42. Dombrowski N, Teske AP, Baker BJ. Expansive microbial metabolic versatility and biodiversity in dynamic Guaymas Basin hydrothermal sediments. Nat Commun. 2018;9(1):4999. https://doi.org/10.1038/s41467-018-07418-0.

43. Dar R, Bandh SA, Shafi S, Shameem N. Bacterial diversity of the rock-water interface in freshwater ecosystem. In: Freshwater Microbiology: Elsevier; 2019. p. 73-104. https://doi.org/10.1016/B978-0-12-817495-1.00002-5.

44. Acinas SG, Sánchez P, Salazar G, et al. Deep ocean metagenomes provide insight into the metabolic architecture of bathypelagic microbial communities. Commun Biol. 2021;4:604. https://doi.org/10.1038/s42003-021-02112-2.

45. Xu Z, Wang M, Wu W, Li Y, Liu Q, Han Y, et al. Vertical distribution of microbial eukaryotes from surface to the hadal zone of the Mariana Trench. Front Microbiol. 2018;9:2023. https://doi.org/10.3389/fmicb.2018.02023.

46. Pernice MC, Giner CR, Logares R, Perera-Bel J, Acinas SG, Duarte CM, et al. Large variability of bathypelagic microbial eukaryotic communities across the world's oceans. ISME J. 2016;10(4):945-58. https://doi.org/10.1038/ismej.2015.170.

47. Wang Z-P, Liu Z-Z, Wang Y-L, Bi W-H, Liu L, Wang H-Y, et al. Fungal community analysis in seawater of the Mariana Trench as estimated by Illumina HiSeq. RSC Adv. 2019;9(12):6956-64. https://doi.org/10.1039/C8RA10142F.

48. Edgcomb VP, Beaudoin D, Gast R, Biddle JF, Teske A. Marine subsurface eukaryotes: the fungal majority. Environ Microbiol. 2011;13(1):172-83. https://doi.org/10.1111/j.1462-2920.2010.02318.x.

49. Jebaraj CS, Raghukumar C, Behnke A, Stoeck T. Fungal diversity in oxygen-depleted regions of the Arabian Sea revealed by targeted environmental sequencing combined with cultivation. FEMS Microbiol Ecol. 2010;71(3):399-412. https://doi. org/10.1111/j.1574-6941.2009.00804.x.

50. Sohlberg E, Bomberg M, Miettinen H, Nyyssönen M, Salavirta H, Vikman M, et al. Revealing the unexplored fungal communities in deep groundwater of crystalline bedrock fracture zones in Olkiluoto, Finland. Front Microbiol. 2015;6: 573.

51. Powell S, Szklarczyk D, Trachana K, Roth A, Kuhn M, Muller J, et al. eggNOG v3. 0: orthologous groups covering 1133 organisms at 41 different taxonomic ranges. Nucleic Acids Res. 2012;40(D1):D284-9. https://doi.org/10.1093/nar/gkr1060. 
52. López-Pérez M, Haro-Moreno JM, Gonzalez-Serrano R, Parras-Moltó M, Rodriguez-Valera F. Genome diversity of marine phages recovered from Mediterranean metagenomes: Size matters. Plos Gen. 2017;13(9):e1007018. https://doi.org/10.13 71/journal.pgen.1007018.

53. Bäckström D, Yutin N, Jørgensen SL, Dharamshi J, Homa F, Zaremba-Niedwiedzka K, et al. Virus genomes from deep sea sediments expand the ocean megavirome and support independent origins of viral gigantism. MBio. 2019;10:e02497-18.

54. Jin M, Guo X, Zhang R, Qu W, Gao B, Zeng R. Diversities and potential biogeochemical impacts of mangrove soil viruses. Microbiome. 2019;7(1):58. https://doi.org/10.1186/s40168-019-0675-9.

55. Roux S, Brum JR, Dutilh BE, Sunagawa S, Duhaime MB, Loy A, et al. Ecogenomics and potential biogeochemical impacts of globally abundant ocean viruses. Nature. 2016;537(7622):689-93. https://doi.org/10.1038/nature19366.

56. Galperin MY, Makarova KS, Wolf YI, Koonin EV. Expanded microbial genome coverage and improved protein family annotation in the COG database. Nucleic Acids Res. 2015;43(D1):D261-9. https://doi.org/10.1093/nar/gku1223.

57. Cantera JJL, Stein LY. Molecular diversity of nitrite reductase genes (nirK) in nitrifying bacteria. Environ Microbiol. 2007; 9(3):765-76. https://doi.org/10.1111/j.1462-2920.2006.01198.x.

58. Margus T, Remm M, Tenson T. Phylogenetic distribution of translational GTPases in bacteria. BMC Genomics. 2007;8(1): 15. https://doi.org/10.1186/1471-2164-8-15

59. Button D, Schut F, Quang P, Martin R, Robertson BR. Viability and isolation of marine bacteria by dilution culture: theory, procedures, and initial results. Appl Environ Microbiol. 1993;59(3):881-91. https://doi.org/10.1128/aem.59.3.881-891.1993.

60. Quast C, Pruesse E, Yilmaz P, Gerken J, Schweer T, Yarza P, et al. The SILVA ribosomal RNA gene database project: improved data processing and web-based tools. Nucleic Acids Res. 2012;41(D1):D590-6. https://doi.org/10.1093/nar/ gks1219.

61. Stackebrandt E, GOEBEL BM. Taxonomic note: a place for DNA-DNA reassociation and 165 rRNA sequence analysis in the present species definition in bacteriology. Int J Syst Evol Microbiol. 1994;44(4):846-9. https://doi.org/10.1099/002 07713-44-4-846.

62. Bolger AM, Lohse M, Usadel B. Trimmomatic: a flexible trimmer for Illumina sequence data. Bioinformatics. 2014;30(15): 2114-20. https://doi.org/10.1093/bioinformatics/btu170.

63. Langmead B, Salzberg SL. Fast gapped-read alignment with Bowtie 2. Nat Methods. 2012;9(4):357-9. https://doi.org/10.1 038/nmeth.1923.

64. Xu H, Luo X, Qian J, Pang X, Song J, Qian G, et al. FastUniq: a fast de novo duplicates removal tool for paired short reads. Plos one. 2012;7(12):e52249. https://doi.org/10.1371/journal.pone.0052249.

65. Li D, Luo R, Liu C-M, Leung C-M, Ting H-F, Sadakane K, et al. MEGAHIT v1. 0: a fast and scalable metagenome assembler driven by advanced methodologies and community practices. Methods. 2016;102:3-11. https://doi.org/10.1016/j.ymeth.2 016.02.020.

66. Alneberg J, Bjarnason BS, De Bruijn I, Schirmer M, Quick J, ljaz UZ, et al. Binning metagenomic contigs by coverage and composition. Nat Methods. 2014;11(11):1144-6. https://doi.org/10.1038/nmeth.3103.

67. Kang D, Froula J, Egan R, Wang Z. MetaBAT: metagenome binning based on abundance and tetranucleotide frequence; 2014.

68. Sieber CM, Probst AJ, Sharrar A, Thomas BC, Hess M, Tringe SG, et al. Recovery of genomes from metagenomes via a dereplication, aggregation and scoring strategy. Nat Microbiol. 2018;3(7):836-43. https://doi.org/10.1038/s41564-018-0171-1.

69. Uritskiy GV, DiRuggiero J, Taylor J. MetaWRAP_-a flexible pipeline for genome-resolved metagenomic data analysis. Microbiome. 2018;6:1-13.

70. Menzel P, Ng KL, Krogh A. Fast and sensitive taxonomic classification for metagenomics with Kaiju. Nat Commun. 2016; 7(1):11257. https://doi.org/10.1038/ncomms11257.

71. Edgar RC. MUSCLE: multiple sequence alignment with high accuracy and high throughput. Nucleic Acids Res. 2004; 32(5):1792-7. https://doi.org/10.1093/nar/gkh340.

72. Capella-Gutiérrez S, Silla-Martínez JM, Gabaldón T. trimAl: a tool for automated alignment trimming in large-scale phylogenetic analyses. Bioinformatics. 2009;25(15):1972-3. https://doi.org/10.1093/bioinformatics/btp348.

73. Stamatakis A. RAxML version 8: a tool for phylogenetic analysis and post-analysis of large phylogenies. Bioinformatics. 2014;30(9):1312-3. https://doi.org/10.1093/bioinformatics/btu033.

74. Letunic I, Bork P. Interactive Tree Of Life (iTOL): an online tool for phylogenetic tree display and annotation. Bioinformatics. 2006;23:127-8.

75. von Meijenfeldt FB, Arkhipova K, Cambuy DD, Coutinho FH, Dutilh BE. Robust taxonomic classification of uncharted microbial sequences and bins with CAT and BAT. Genome Biol. 2019;20(1):217. https:/doi.org/10.1186/s13059-019-1817-X.

76. Hyatt D, Chen G-L, LoCascio PF, Land ML, Larimer FW, Hauser LJ. Prodigal: prokaryotic gene recognition and translation initiation site identification. BMC Bioinformatics. 2010;11(1):119. https://doi.org/10.1186/1471-2105-11-119.

77. Moriya Y, Itoh M, Okuda S, Yoshizawa AC, Kanehisa M. KAAS: an automatic genome annotation and pathway reconstruction server. Nucleic Acids Res. 2007;35(Web Server):W182-5. https://doi.org/10.1093/nar/gkm321.

78. Yin Y, Mao X, Yang J, Chen X, Mao F, Xu Y. dbCAN: a web resource for automated carbohydrate-active enzyme annotation. Nucleic acids Res. 2012;40(W1):W445-51. https://doi.org/10.1093/nar/gks479.

79. Adriaenssens EM, Cowan DA. Using signature genes as tools to assess environmental viral ecology and diversity. Appl Environ Microbiol. 2014;80(15):4470-80. https://doi.org/10.1128/AEM.00878-14.

80. Katoh K, Toh H. Parallelization of the MAFFT multiple sequence alignment program. Bioinformatics. 2010;26(15):1899900. https://doi.org/10.1093/bioinformatics/btq224.

81. Price MN, Dehal PS, Arkin AP. FastTree 2-approximately maximum-likelihood trees for large alignments. Plos one. 2010; 5(3):e9490. https://doi.org/10.1371/journal.pone.0009490.

82. Bland C, Ramsey TL, Sabree F, Lowe M, Brown K, Kyrpides NC, et al. CRISPR recognition tool (CRT): a tool for automatic detection of clustered regularly interspaced palindromic repeats. BMC Bioinformatics. 2007;8(1):209. https://doi.org/10.11 86/1471-2105-8-209.

83. Levasseur A, Drula E, Lombard V, Coutinho PM, Henrissat B. Expansion of the enzymatic repertoire of the CAZy database to integrate auxiliary redox enzymes. Biotechnol Biofuels. 2013;6(1):41. https://doi.org/10.1186/1754-6834-6-41.

84. Rappé MS, Connon SA, Vergin KL, Giovannoni SJ. Cultivation of the ubiquitous SAR11 marine bacterioplankton clade. Nature. 2002;418(6898):630-3. https://doi.org/10.1038/nature00917. 
85. Toju H, Tanabe AS, Yamamoto S, Sato H. High-coverage ITS primers for the DNA-based identification of ascomycetes and basidiomycetes in environmental samples. Plos one. 2012;7(7):e40863. https://doi.org/10.1371/journal.pone.0040863.

86. Song $T$, Chen $M$, Chai $W$, Zhang Z, Lian X-Y. New bioactive pyrrospirones $C-I$ from a marine-derived fungus Penicillium sp. ZZ380. Tetrahedron. 2018;74(8):884-91. https://doi.org/10.1016/j.tet.2018.01.015.

87. Terashima M, Kamagata Y, Kato S. Rapid enrichment and isolation of polyphosphate-accumulating organisms through 4'6-diamidino-2-phenylindole (DAPI) staining with fluorescence-activated cell sorting (FACS). Front Microbiol. 2020;11. https://doi.org/10.3389/fmicb.2020.00793.

88. Hageage GJ, Harrington BJ. Use of Calcofluor White in clinical mycology. Labmedicine. 1984;15:109-12.

89. Chen P, Zhou H, Huang YY, Xie Z, Zhang MJ, Wei YL, Li J, Ma YW, Luo M, Ding WM, Cao JW, Jiang T, Nan P, Fang JS, Li X. Mariana Trench Sediment Metagenome. NCBI BioProject PRJNA723166. https://www.ncbi.nlm.nih.gov/bioproject/ PRJNA723166. Accessed 06 June 2021.

\section{Publisher's Note}

Springer Nature remains neutral with regard to jurisdictional claims in published maps and institutional affiliations.

Ready to submit your research? Choose BMC and benefit from:

- fast, convenient online submission

- thorough peer review by experienced researchers in your field

- rapid publication on acceptance

- support for research data, including large and complex data types

- gold Open Access which fosters wider collaboration and increased citations

- maximum visibility for your research: over $100 \mathrm{M}$ website views per year

At $\mathrm{BMC}$, research is always in progress. 